\title{
Stereoselective total synthesis of (+)-cardiobutanolide
}

Kavirayani R. Prasad* and Shivajirao L. Gholap

Department of Organic Chemistry, Indian Institute of Science, Bangalore 560012, India e-mail:prasad@orgchem.iisc.ernet.in

\section{Supplementary information}

\begin{tabular}{|c|c|}
\hline S2 & $:{ }^{1} \mathrm{H}$ NMR Spectrum of compound 4 \\
\hline S3 & $:{ }^{13} \mathrm{C}$ NMR Spectrum of compound 4 \\
\hline S4 & $:{ }^{1} \mathrm{H}$ NMR Spectrum of compound 5 \\
\hline 5 & $:{ }^{13} \mathrm{C}$ NMR Spectrum of compound 5 \\
\hline 6 & $:{ }^{1} \mathrm{H}$ NMR Spectrum of compound 6 \\
\hline S7 & $:{ }^{13} \mathrm{C}$ NMR Spectrum of compound 6 \\
\hline S8 & $:{ }^{1} \mathrm{H}$ NMR Spectrum of compound 7 \\
\hline S9 & $:{ }^{13} \mathrm{C}$ NMR Spectrum of compound 7 \\
\hline S10 & $:{ }^{1} \mathrm{H}$ NMR Spectrum of compound 8 \\
\hline 11 & $:{ }^{13} \mathrm{C}$ NMR Spectrum of compound 8 \\
\hline 12 & : ${ }^{1} \mathrm{H}$ NMR Spectrum of compound 9 \\
\hline 13 & $:{ }^{13} \mathrm{C}$ NMR Spectrum of compound 9 \\
\hline 514 & $:{ }^{1} \mathrm{H}$ NMR Spectrum of compound $\mathbf{1 0}$ \\
\hline 15 & $:{ }^{13} \mathrm{C}$ NMR Spectrum of compound $\mathbf{1 0}$ \\
\hline 16 & $:{ }^{1} \mathrm{H}$ NMR Spectrum of compound $\mathbf{1 1}$ \\
\hline S17 & $:{ }^{13} \mathrm{C}$ NMR Spectrum of compound $\mathbf{1 1}$ \\
\hline S18 & : ${ }^{1} \mathrm{H}$ NMR Spectrum of compound $\mathbf{1 2} \& \mathbf{1 3}$ \\
\hline S19 & $:{ }^{13} \mathrm{C}$ NMR Spectrum of compound $12 \& 13$ \\
\hline 20 & $:{ }^{1} \mathrm{H}$ NMR Spectrum of compound $\mathbf{1 4} \& \mathbf{1 5}$ \\
\hline 521 & $:{ }^{13} \mathrm{C}$ NMR Spectrum of compound $\mathbf{1 4} \& 15$ \\
\hline S22 & $:{ }^{1} \mathrm{H}$ NMR Spectrum of compound 16 \\
\hline S23 & $:{ }^{13} \mathrm{C}$ NMR Spectrum of compound $\mathbf{1 6}$ \\
\hline S24 & $:{ }^{1} \mathrm{H}$ NMR Spectrum of compound $\mathbf{1 7}$ \\
\hline S25 & $:{ }^{13} \mathrm{C}$ NMR Spectrum of compound $\mathbf{1 7}$ \\
\hline S26 & $:{ }^{1} \mathrm{H}$ NMR Spectrum of compound $\mathbf{1}$ \\
\hline S27 & $:{ }^{13} \mathrm{C}$ NMR Spectrum of compound $\mathbf{1}$ \\
\hline S28 & $:{ }^{1} \mathrm{H}$ NMR Spectrum of compound 2 \\
\hline S29 & $:{ }^{13} \mathrm{C}$ NMR Spectrum of compound 2 \\
\hline
\end{tabular}




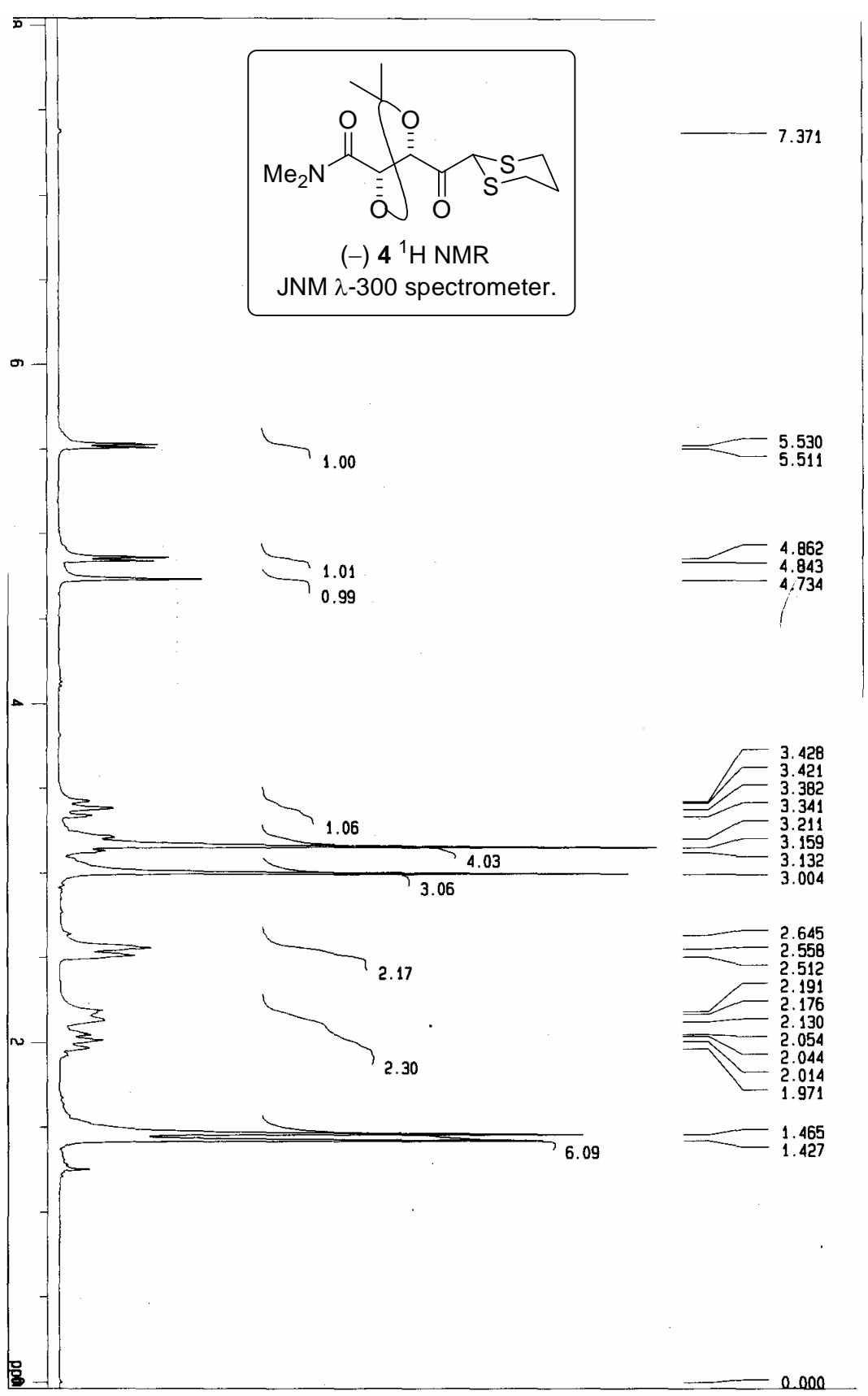




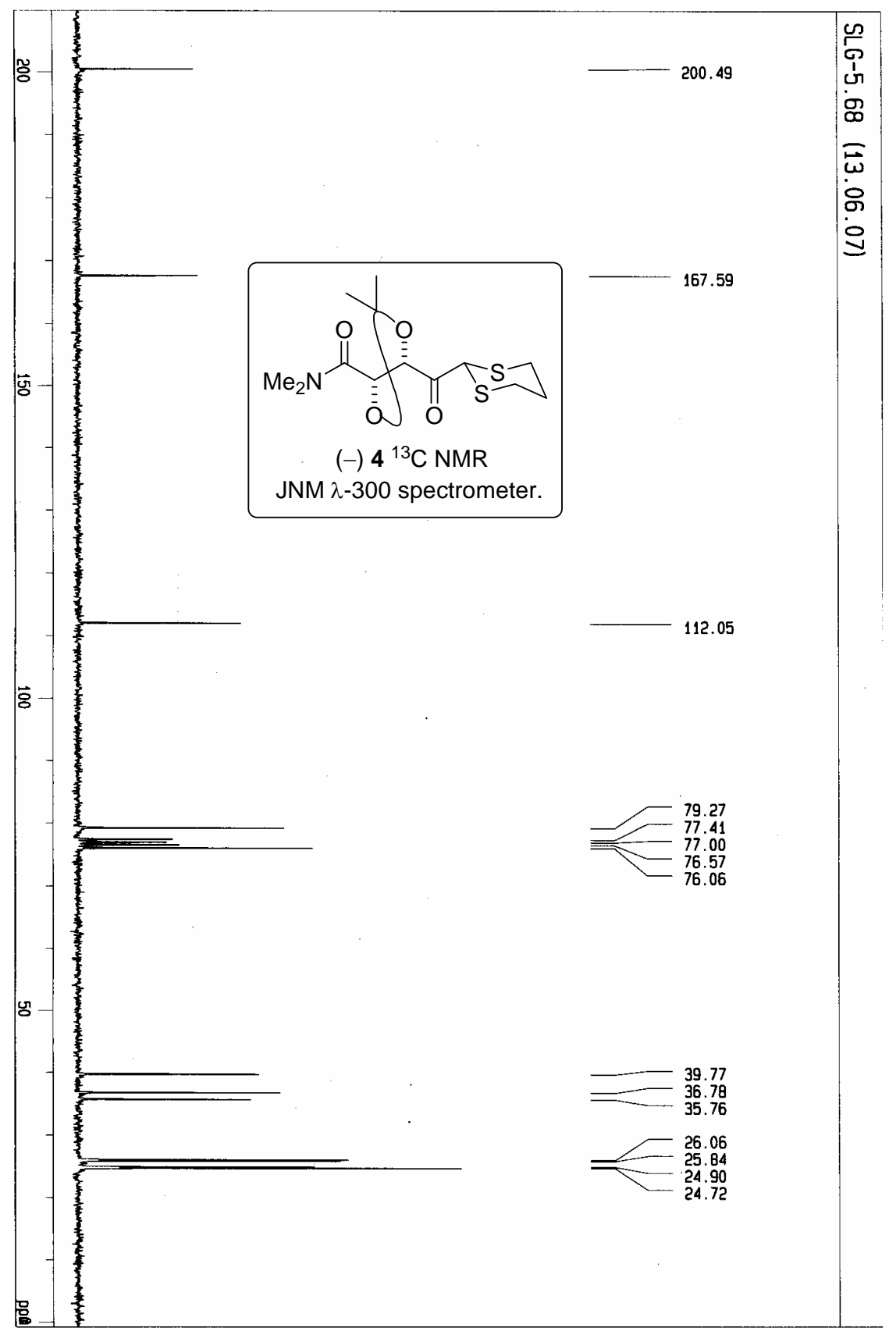




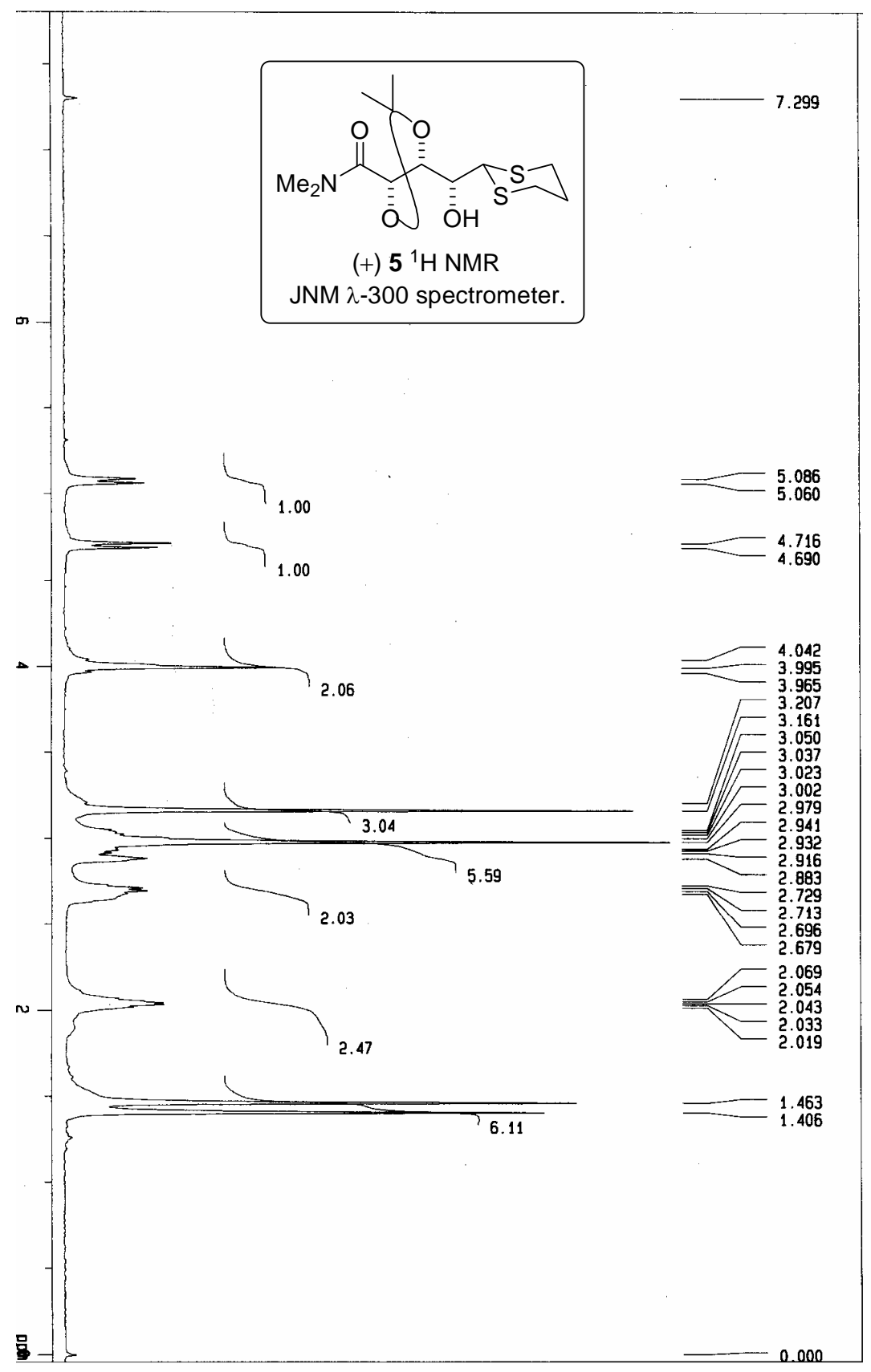




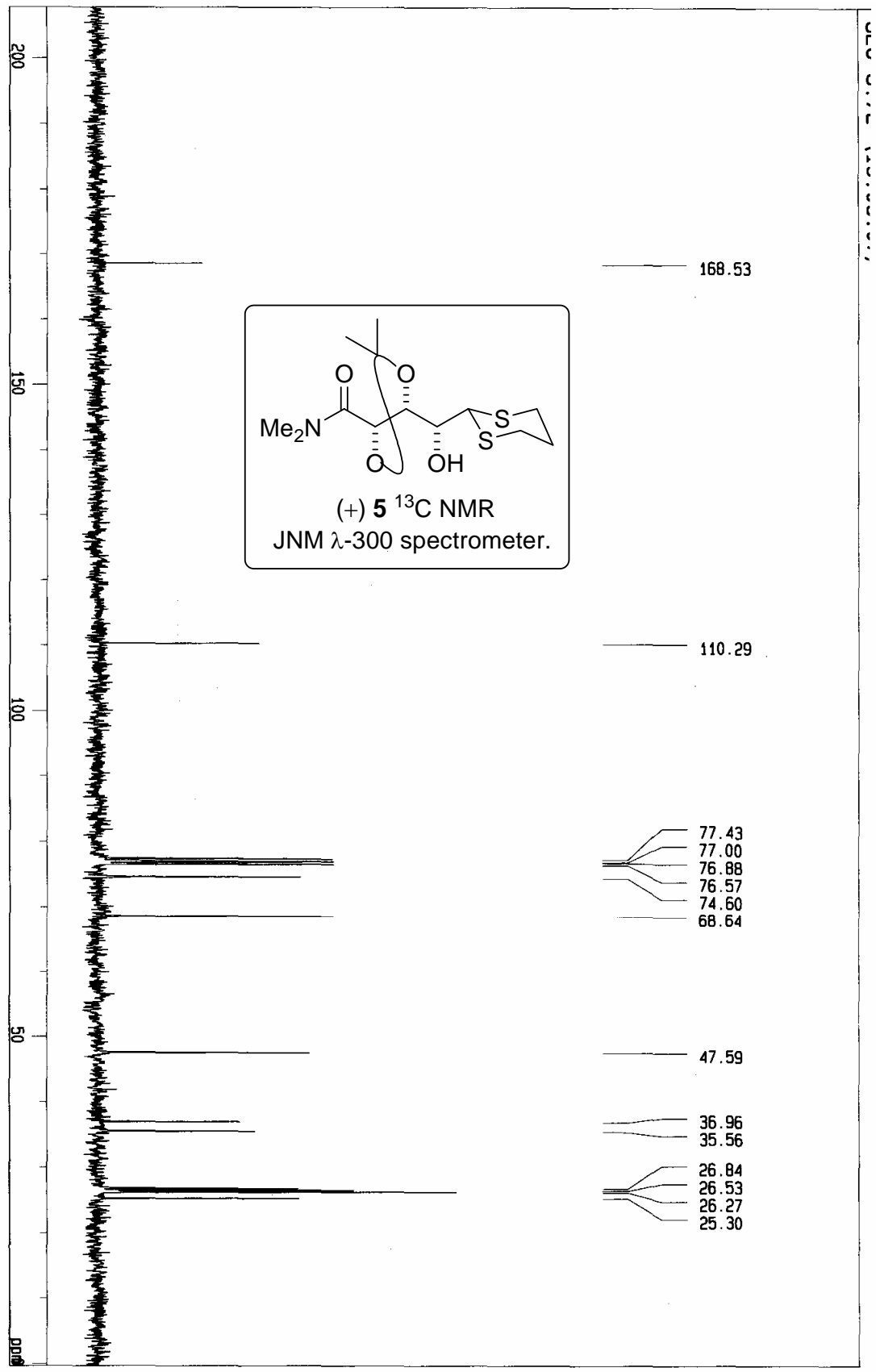




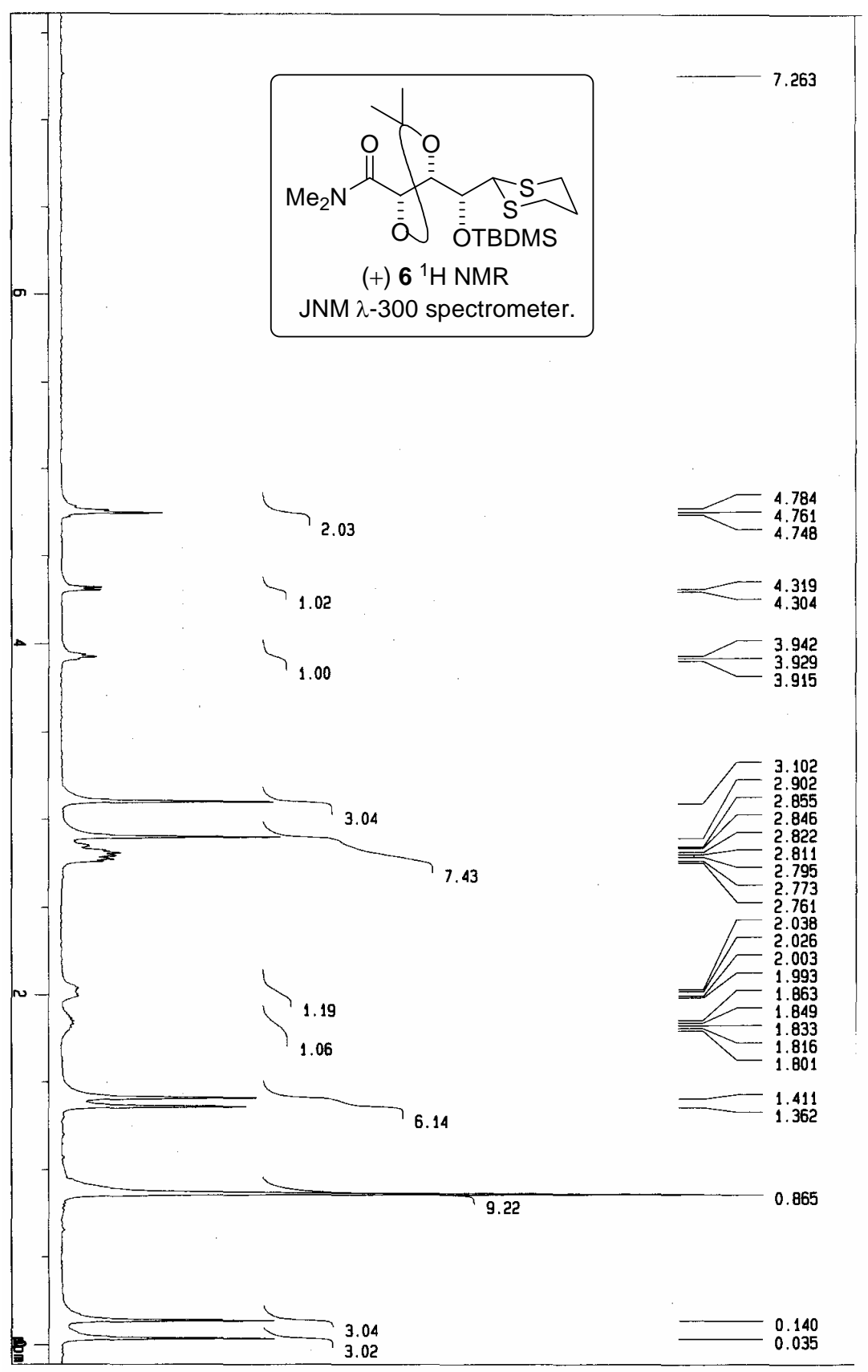




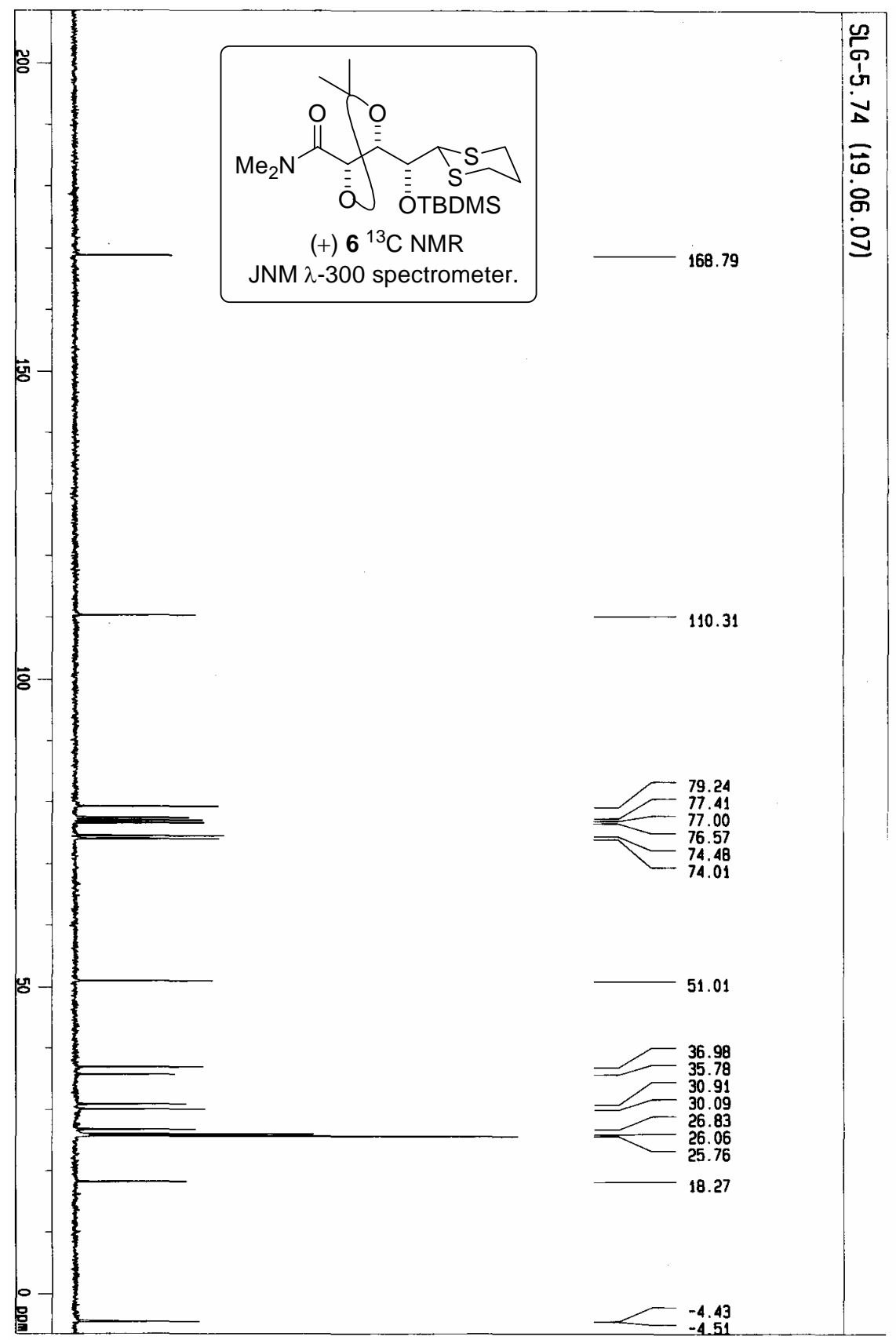




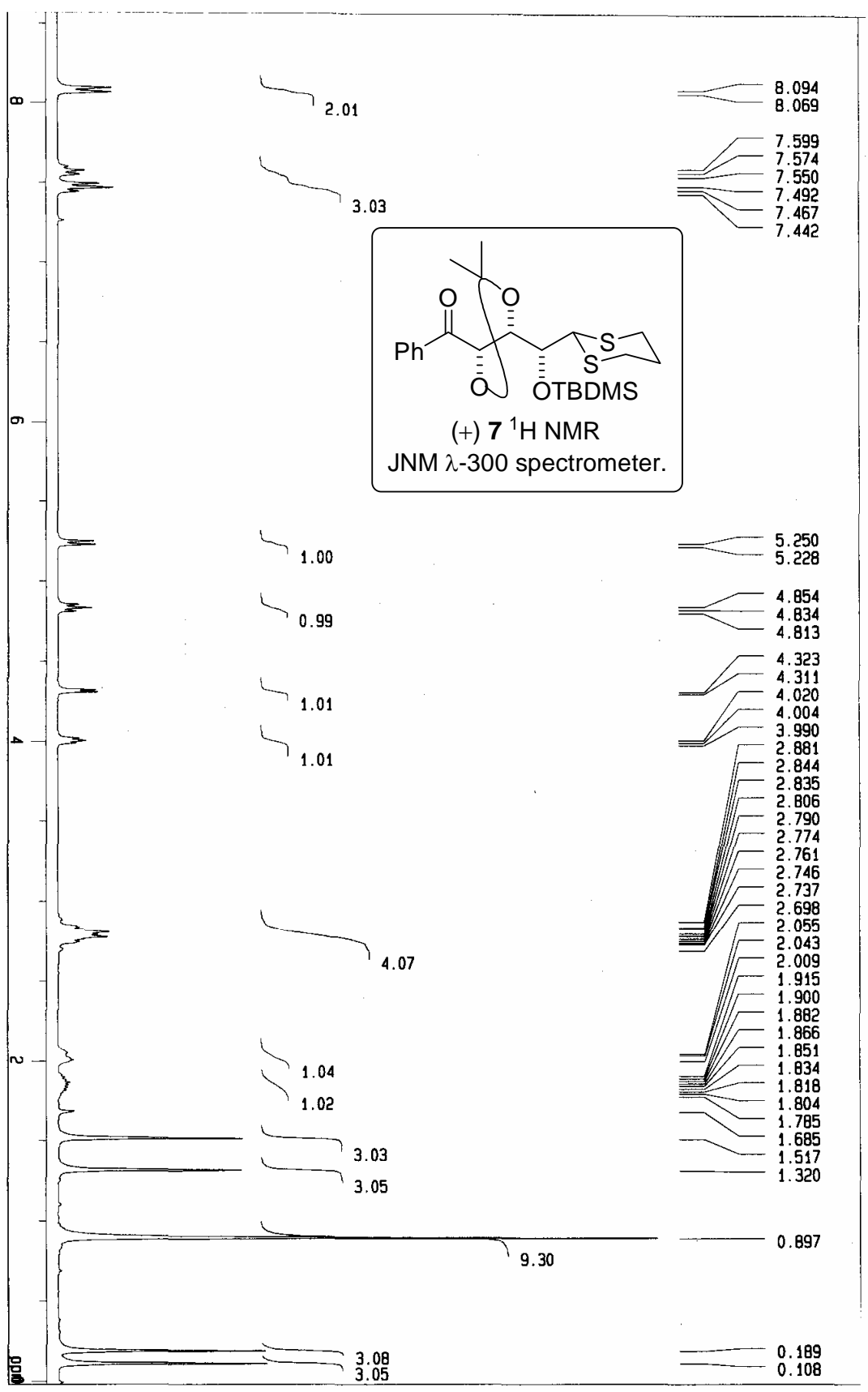




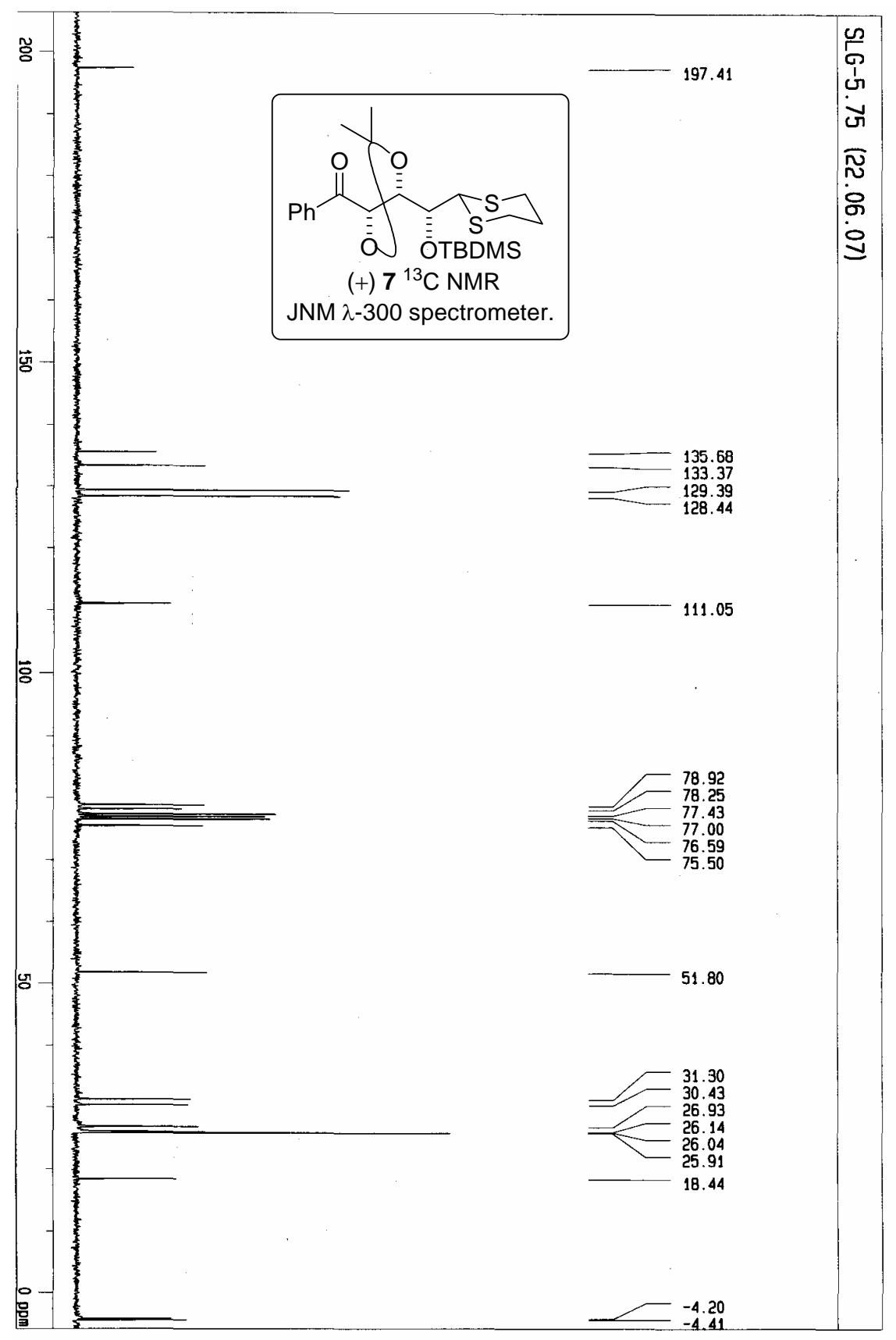




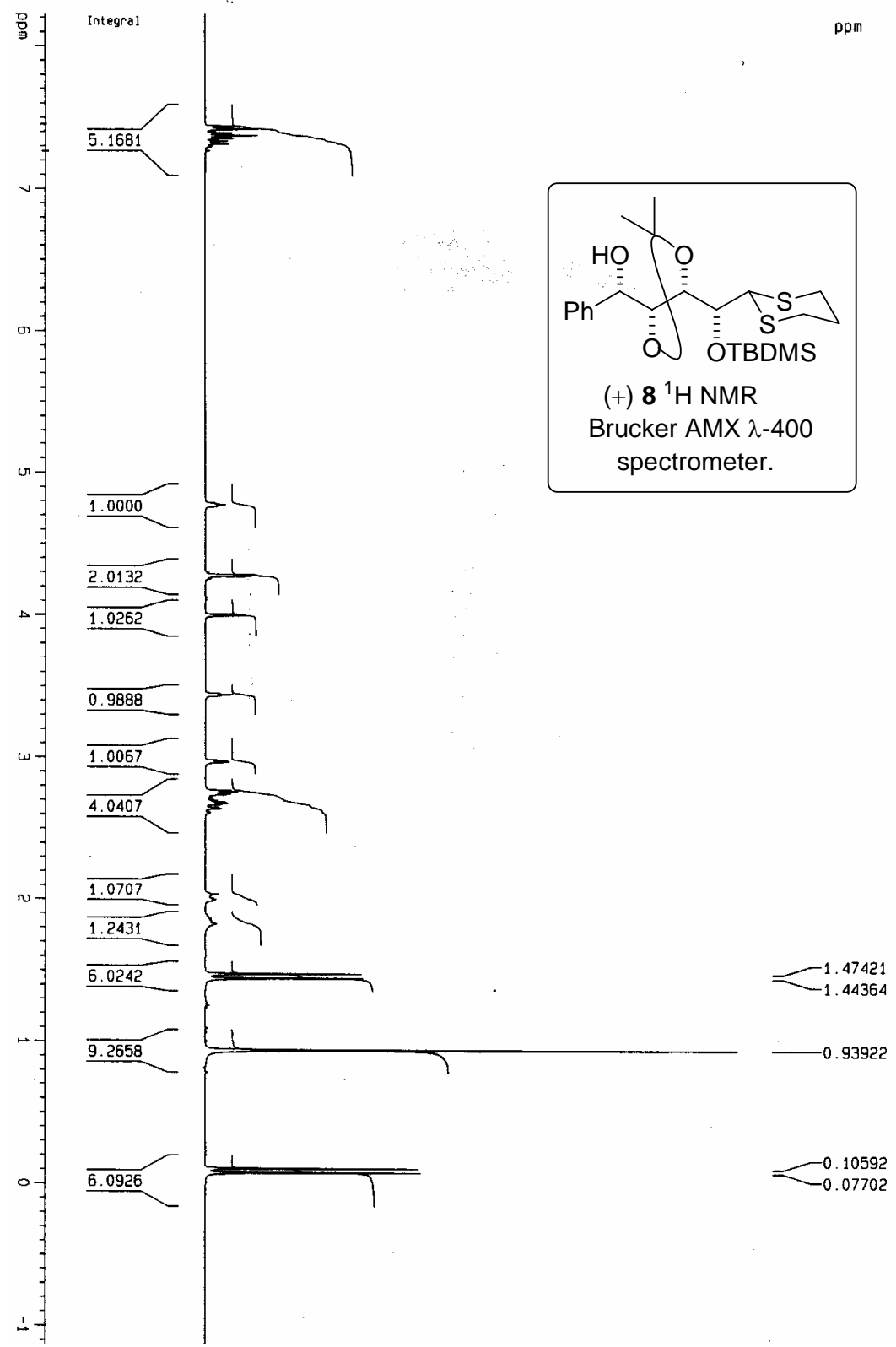



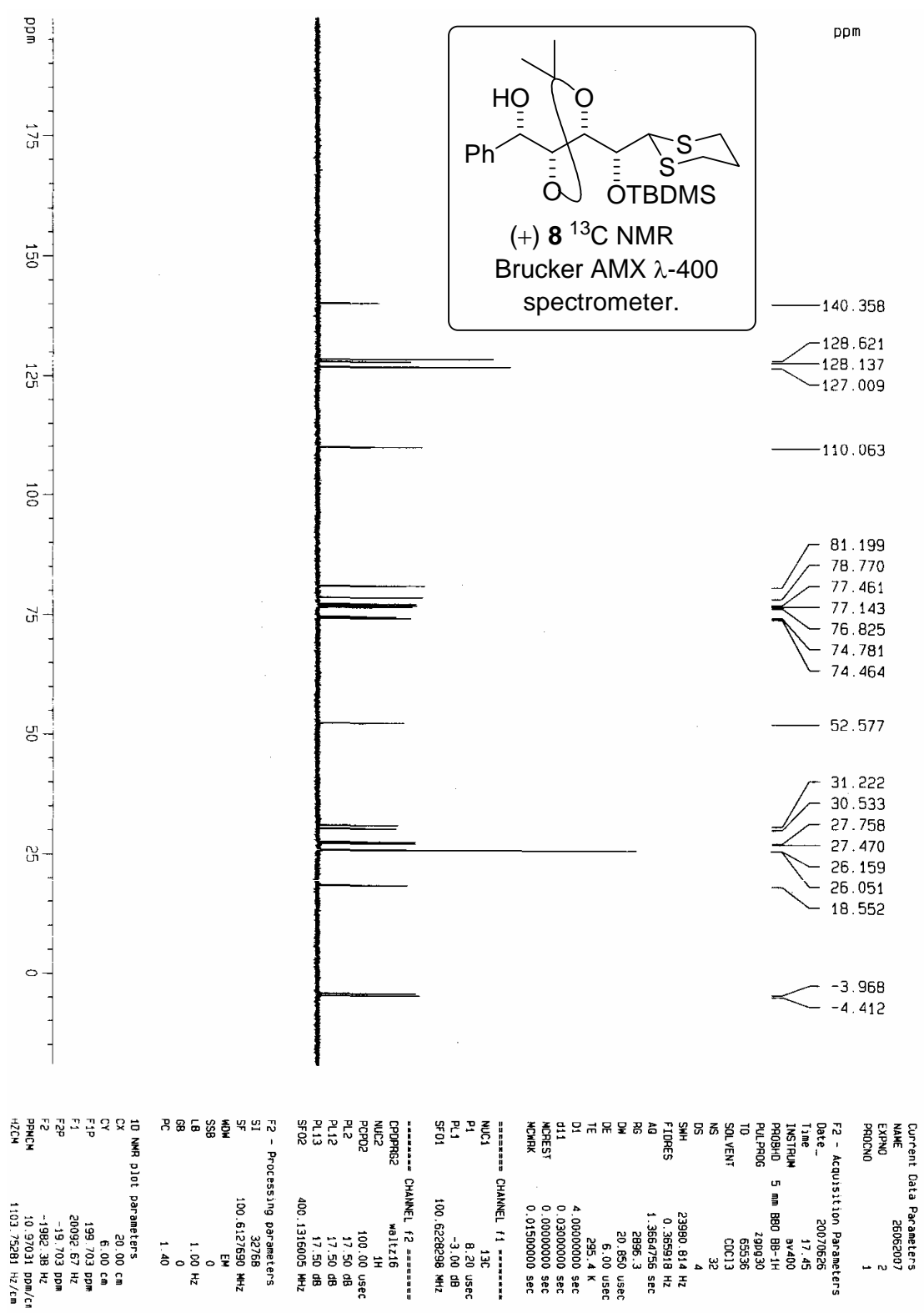


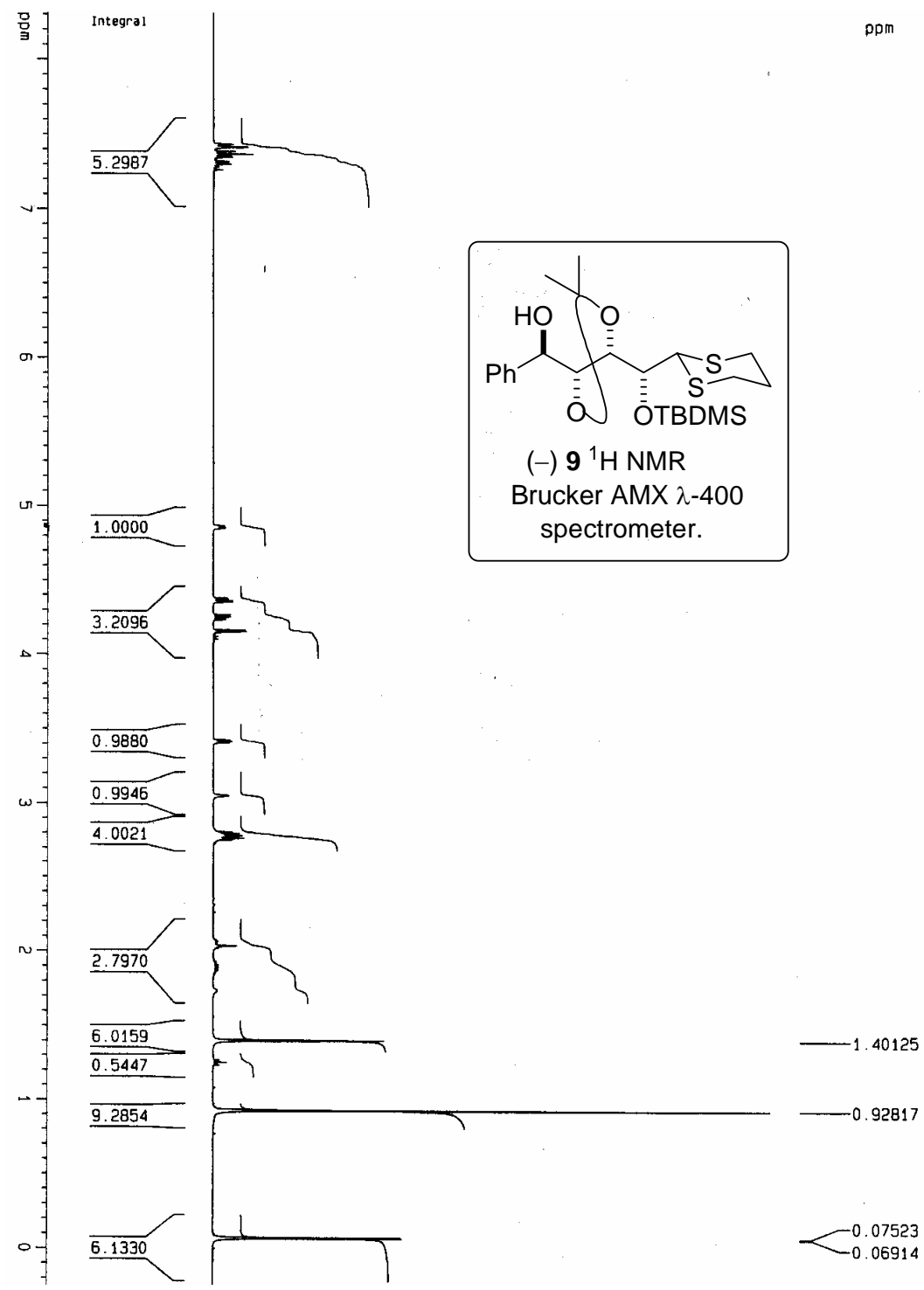



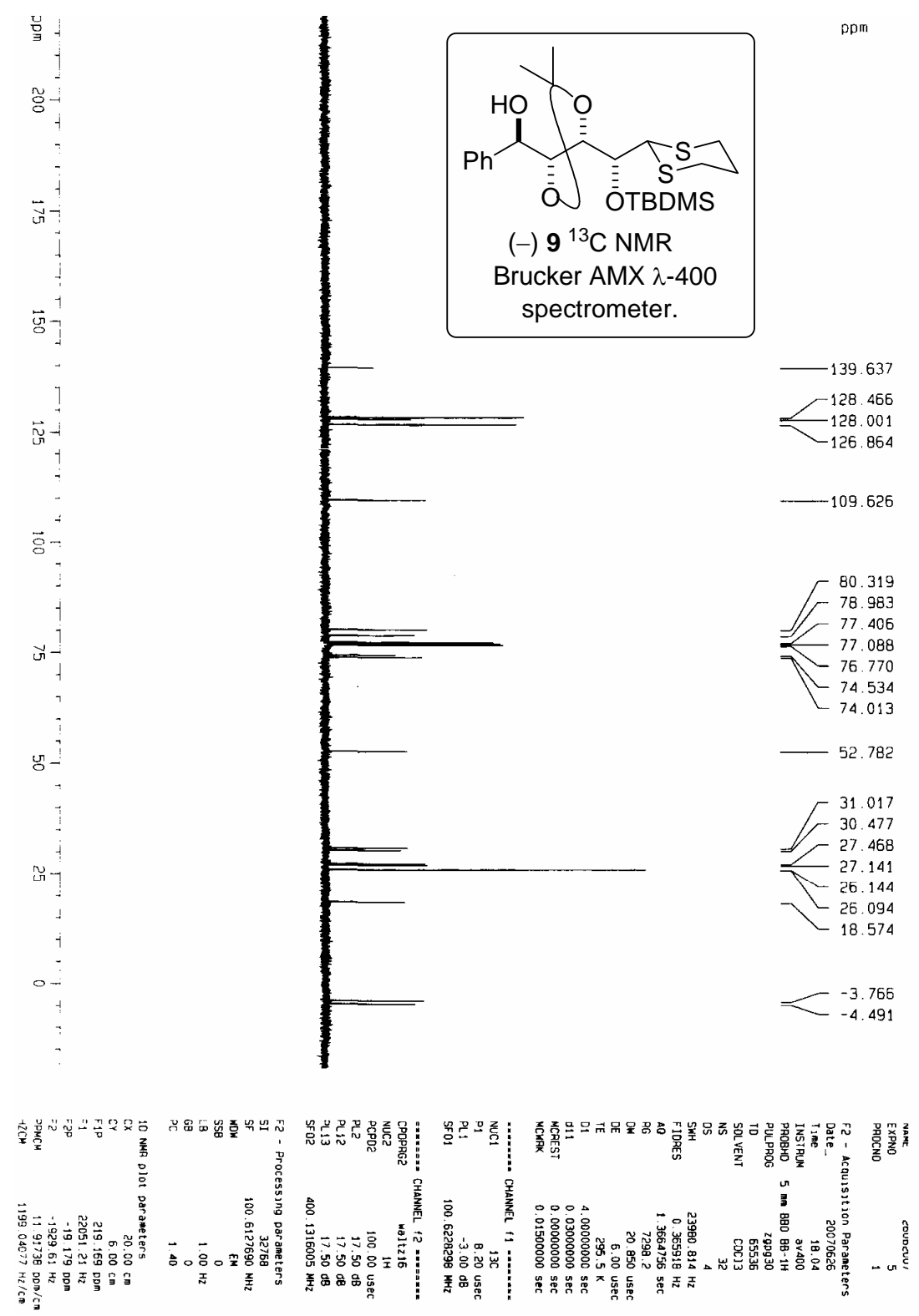


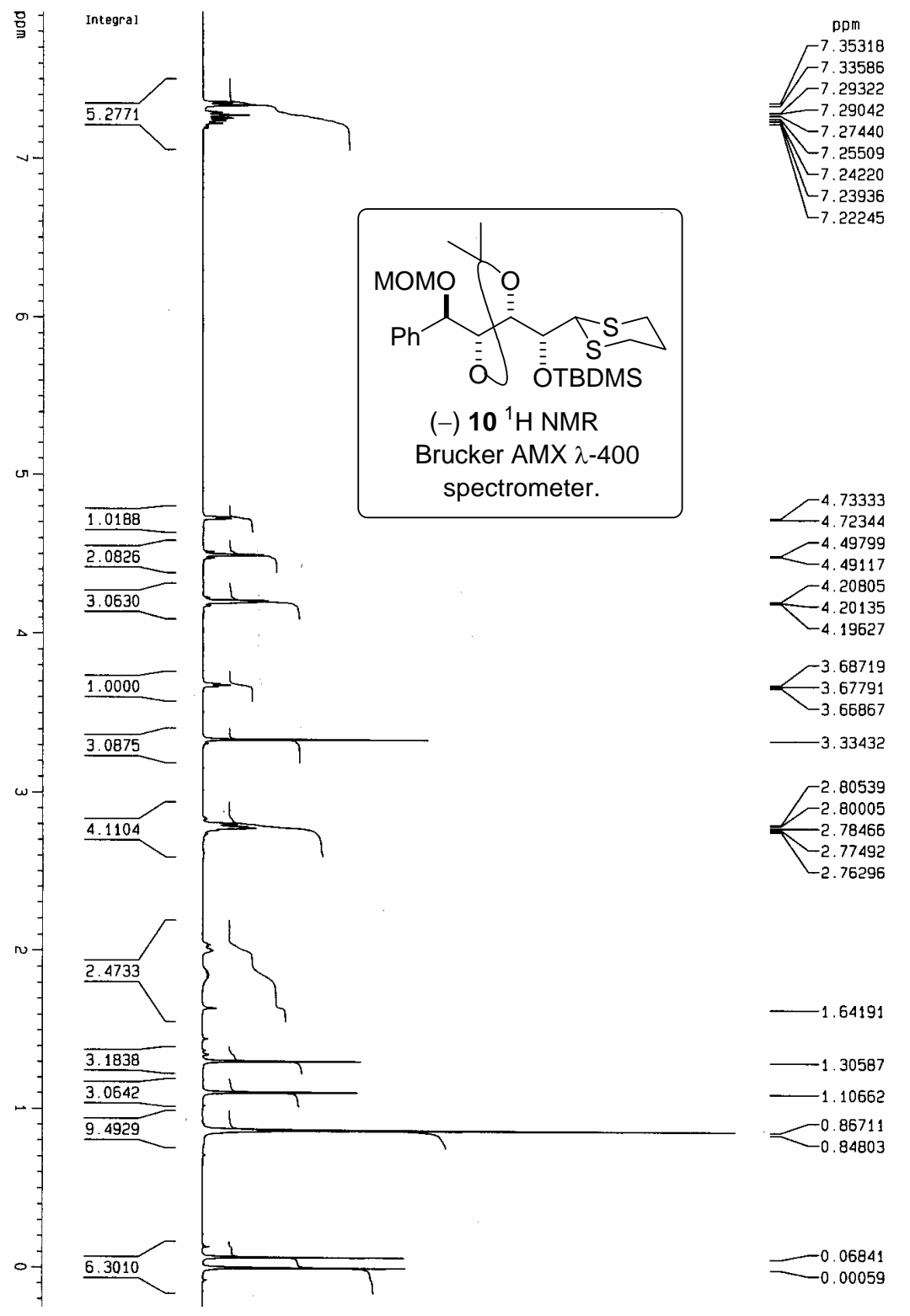



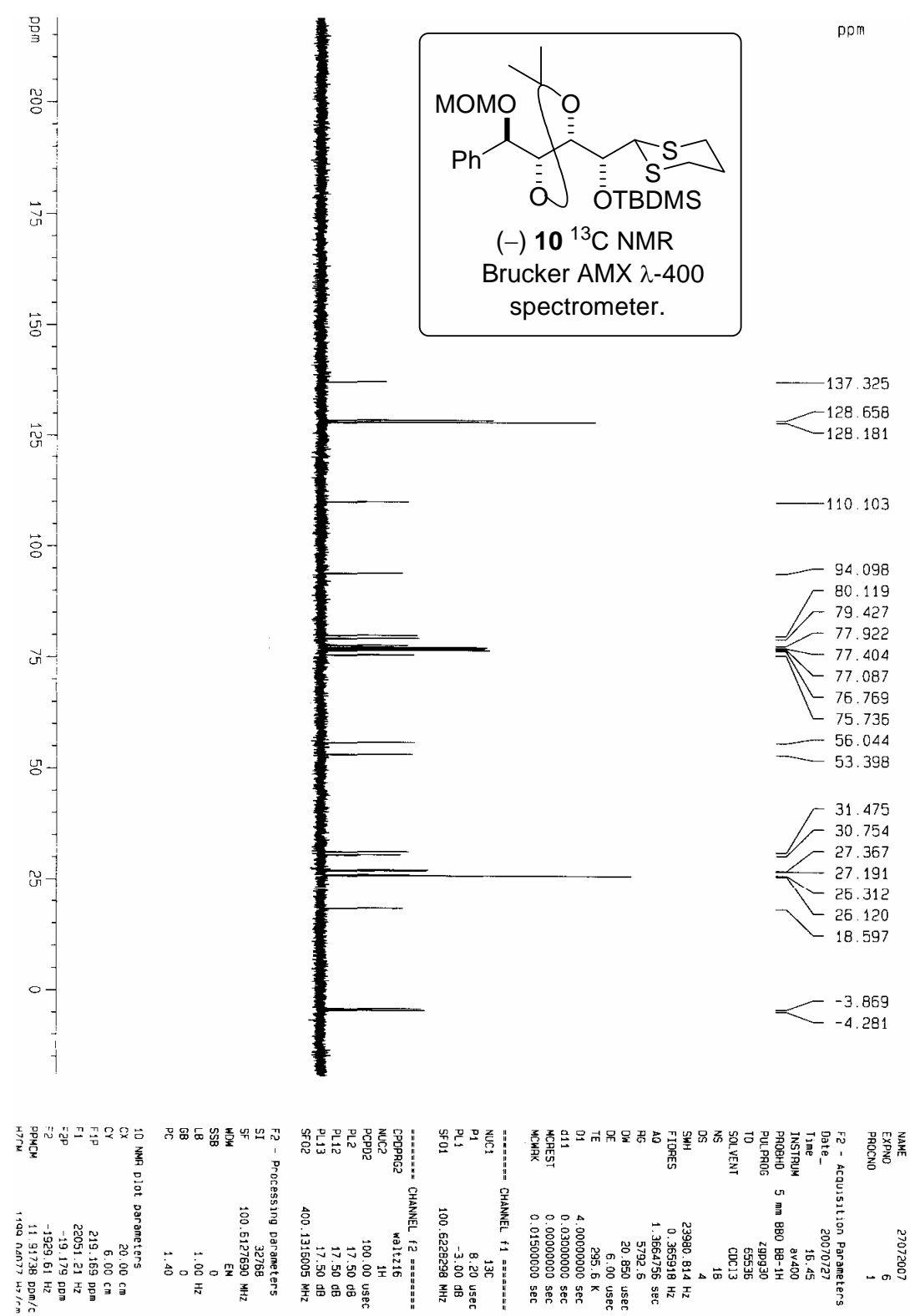


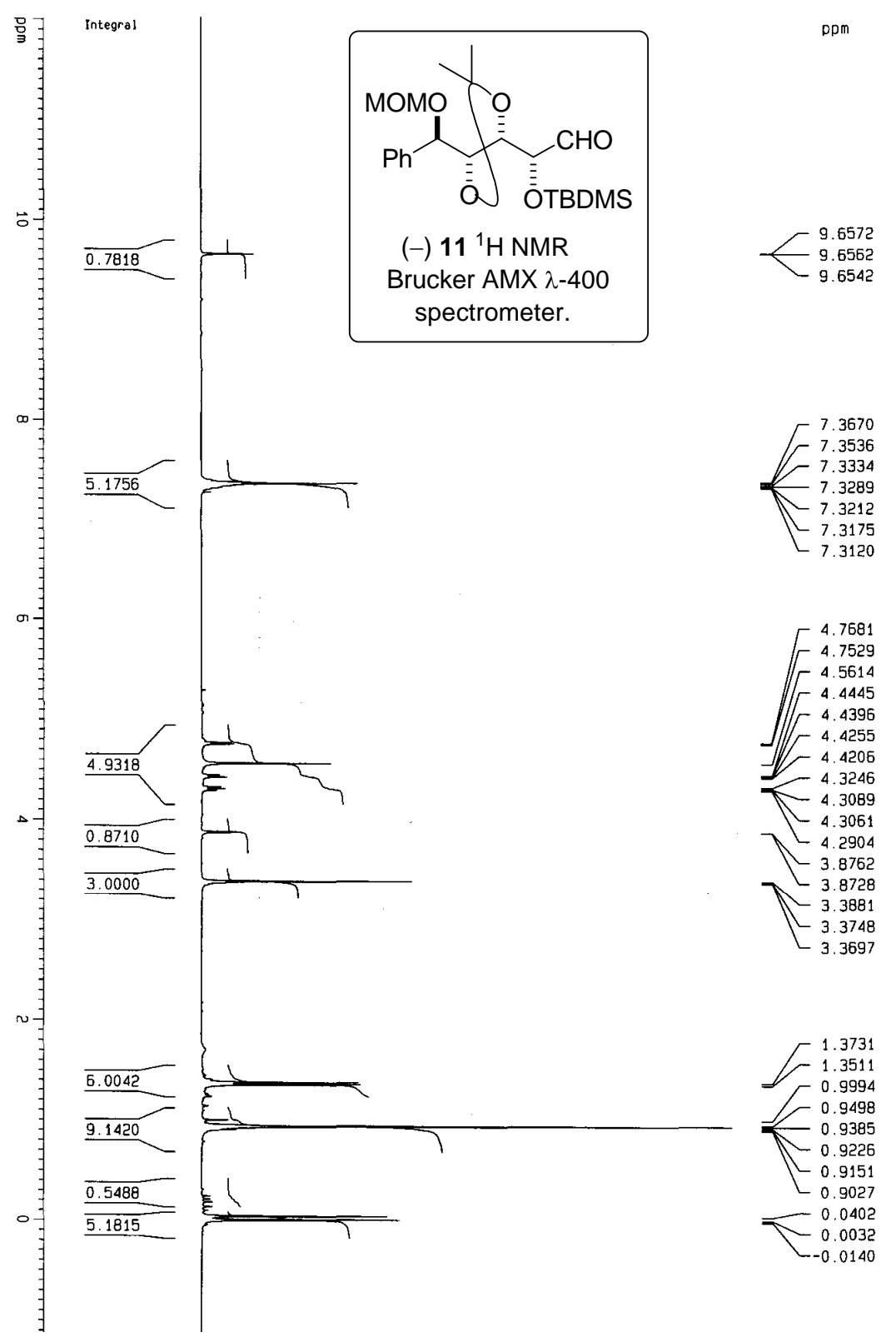



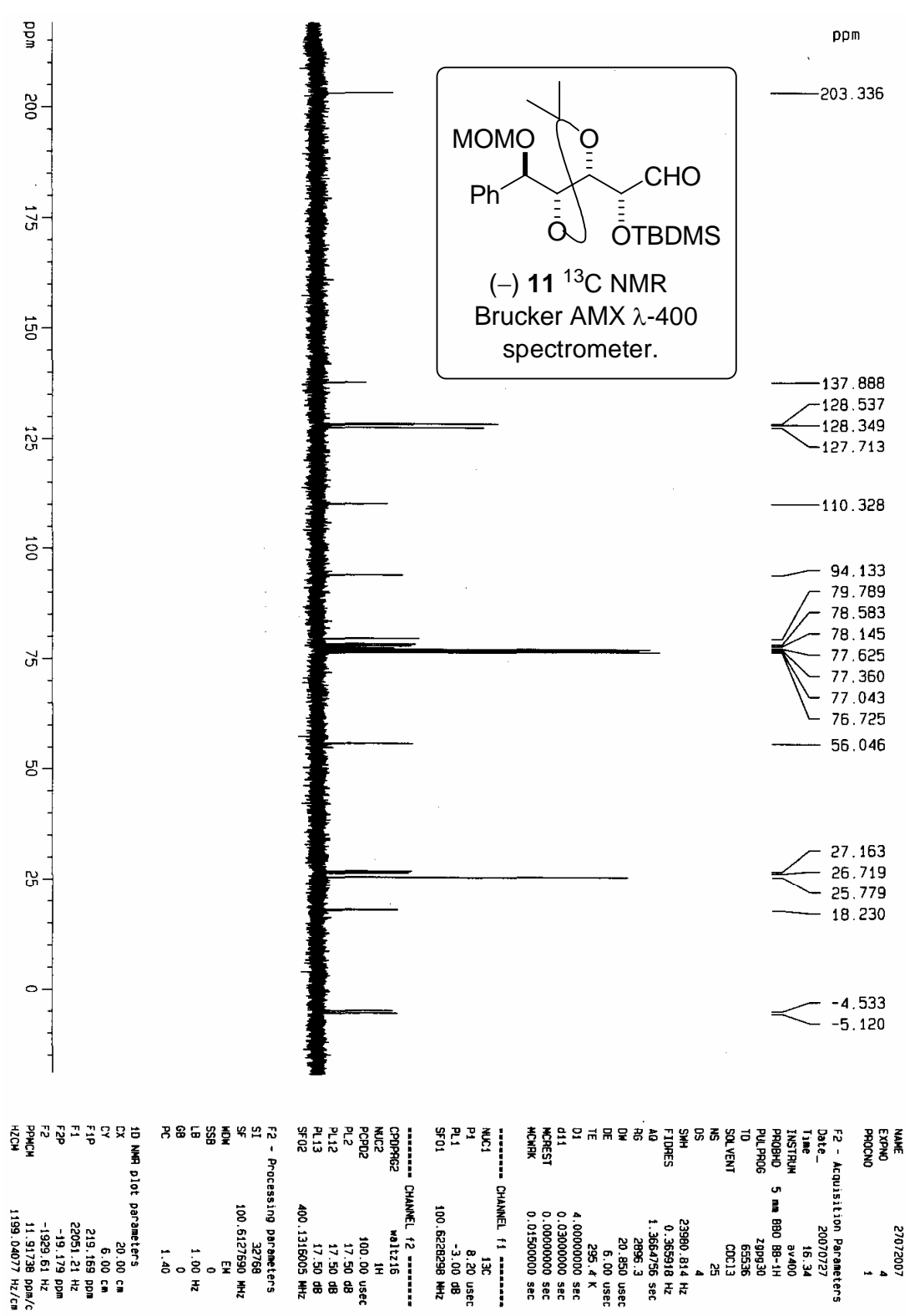


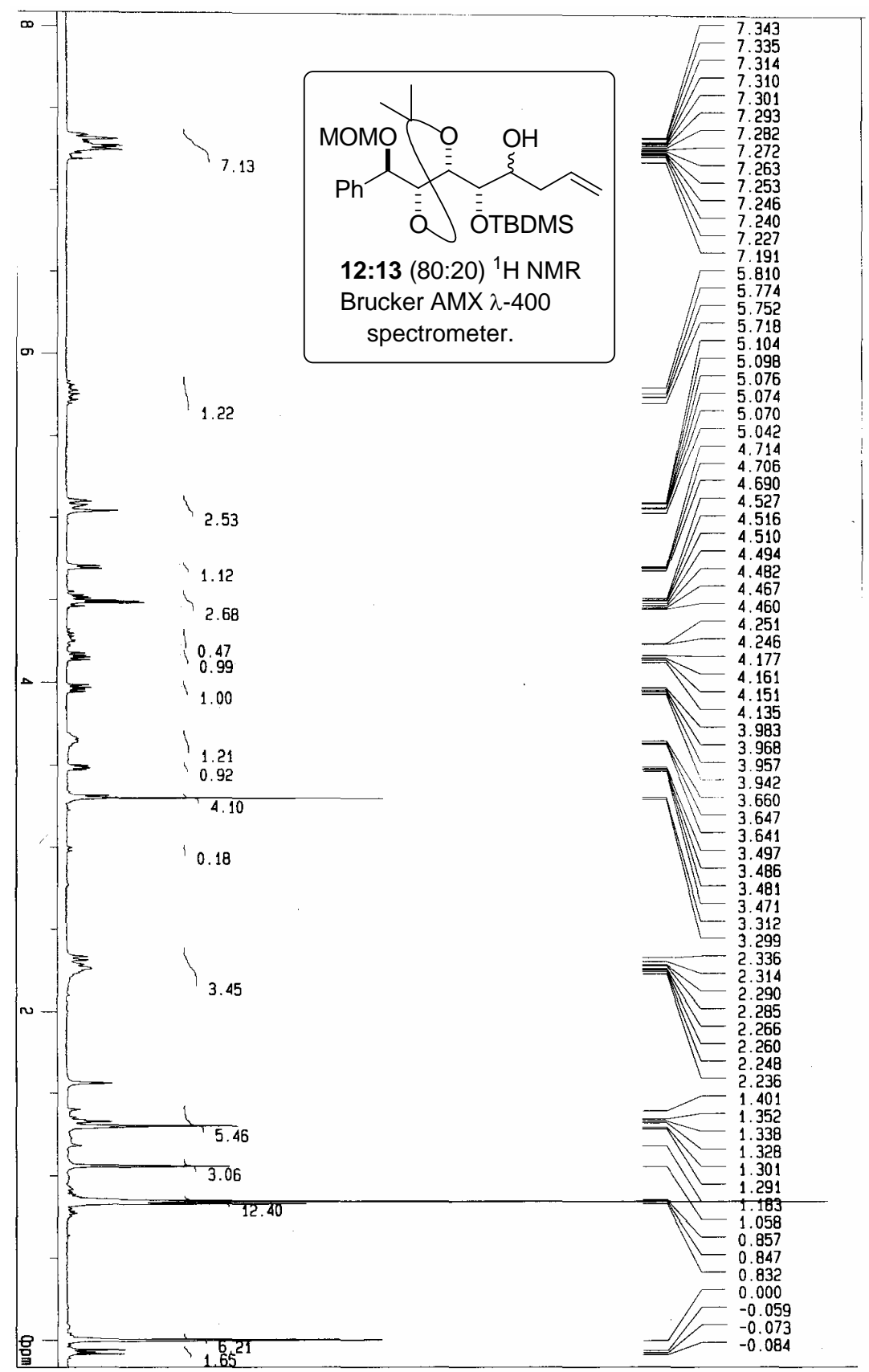




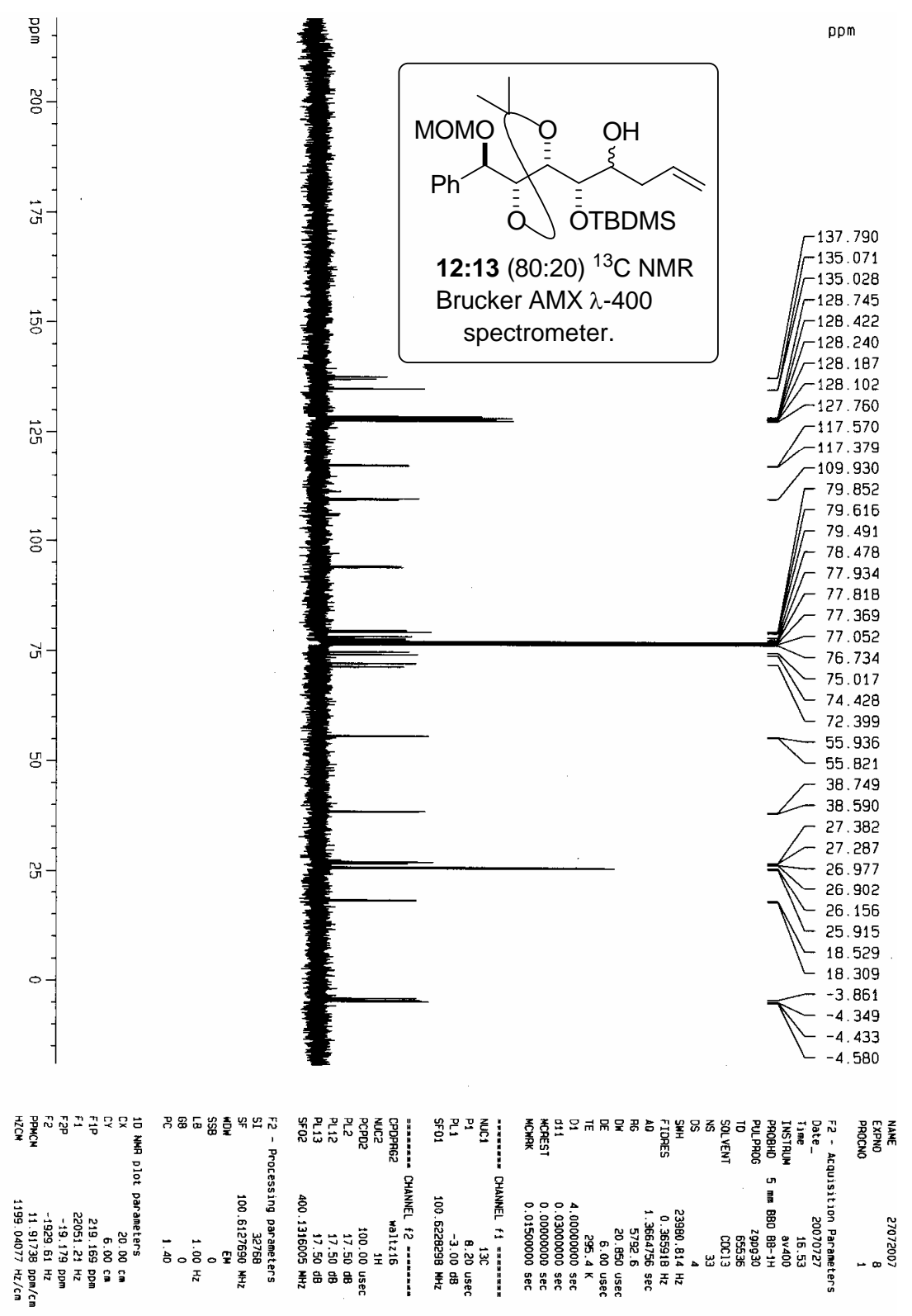




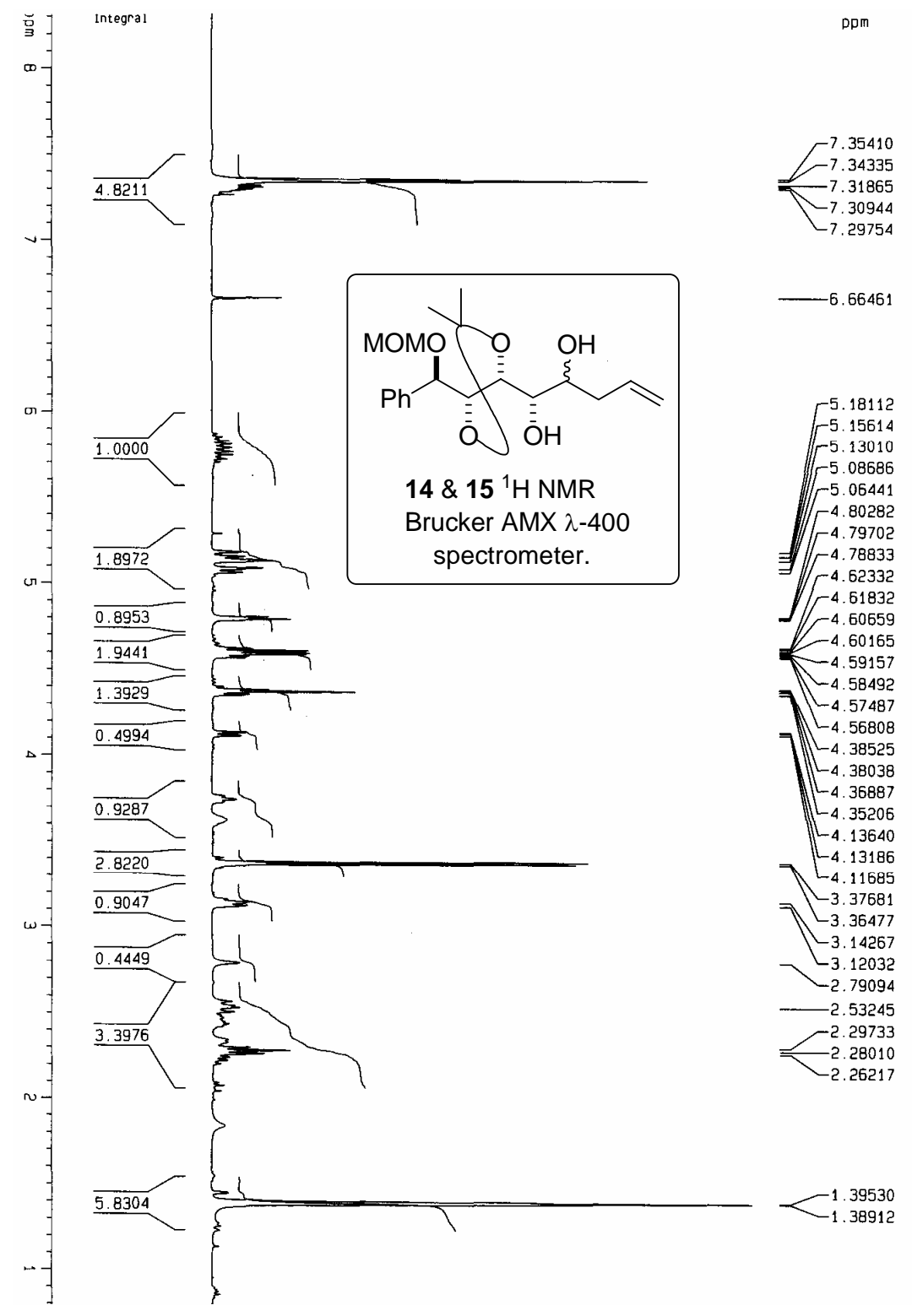



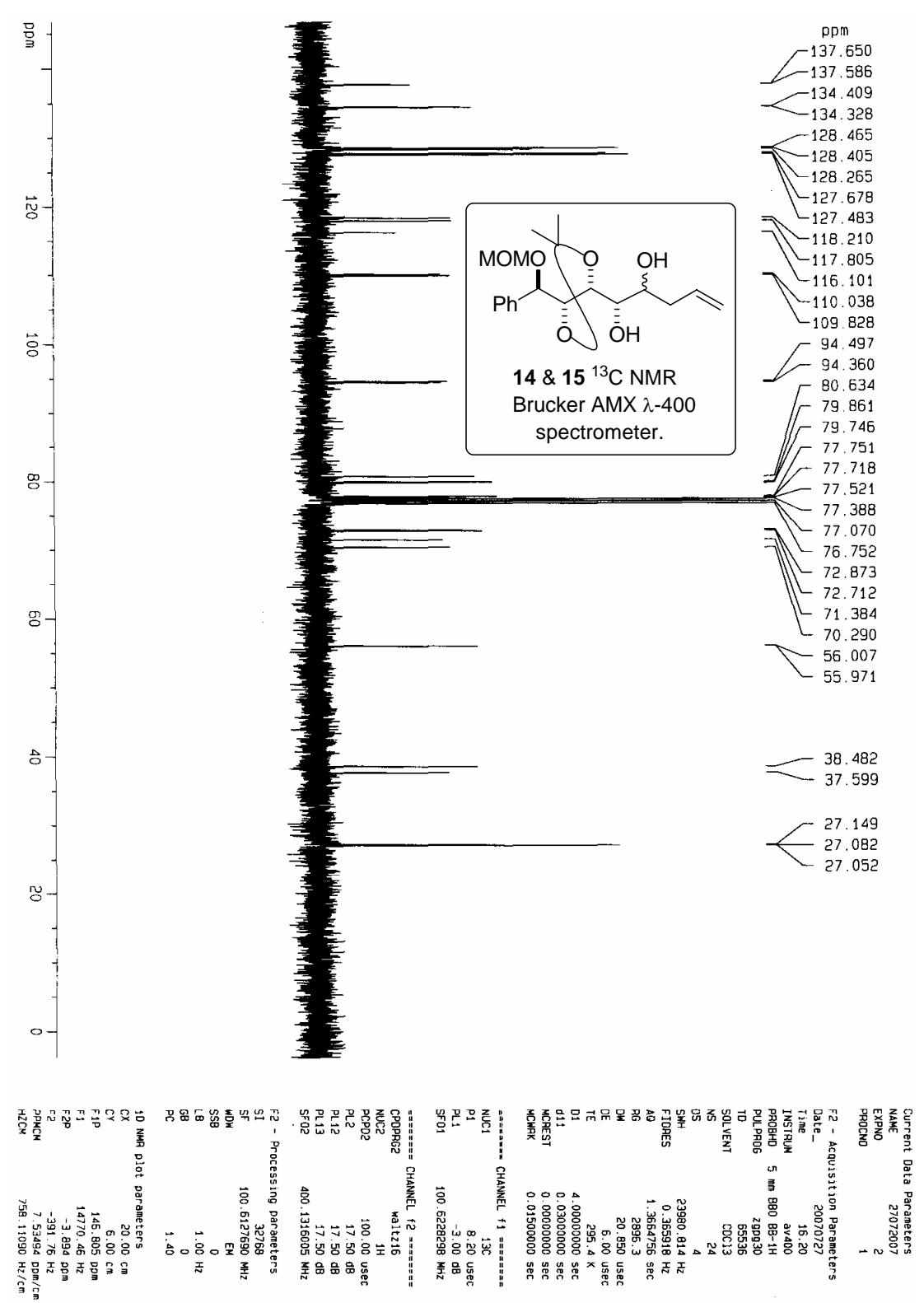


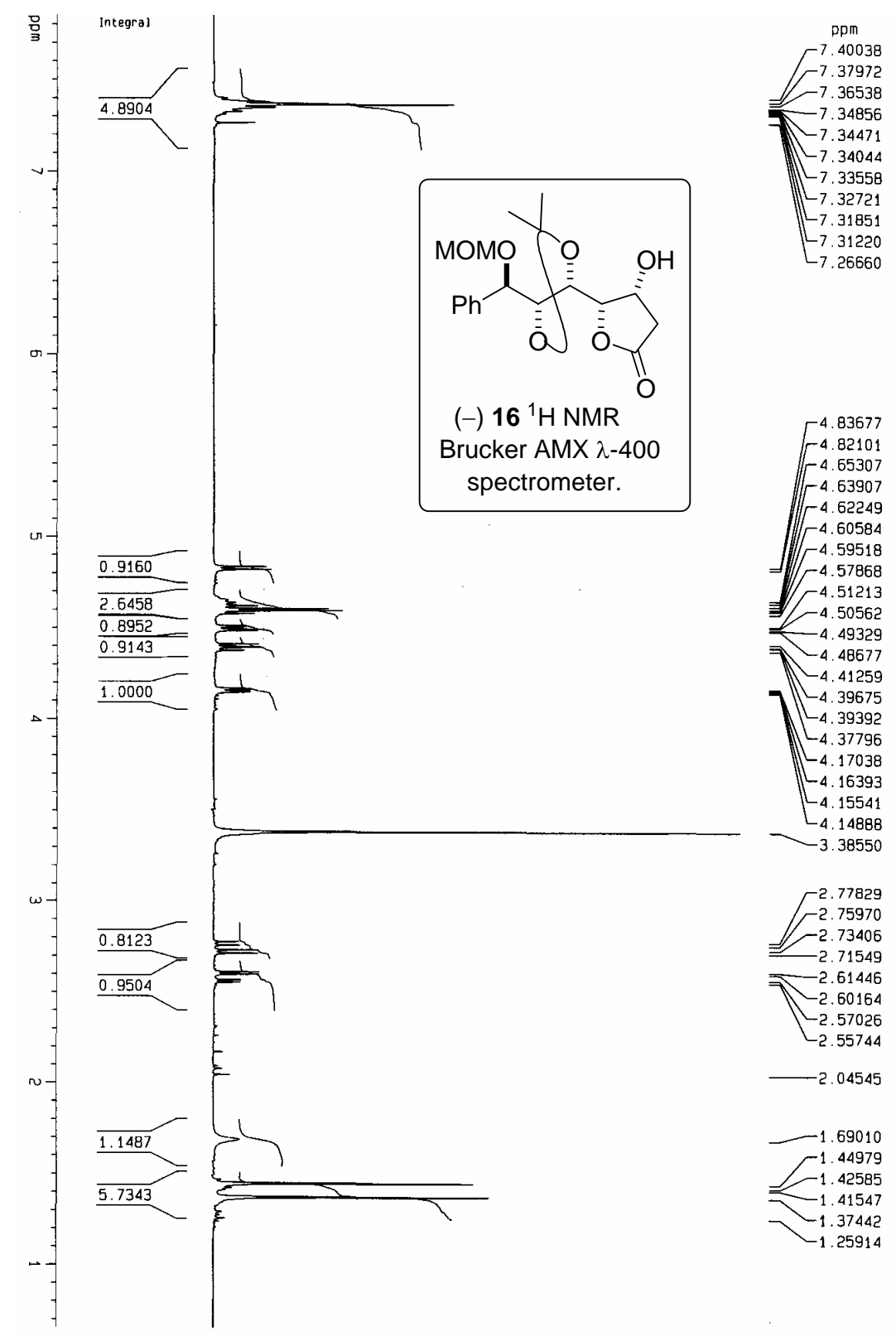




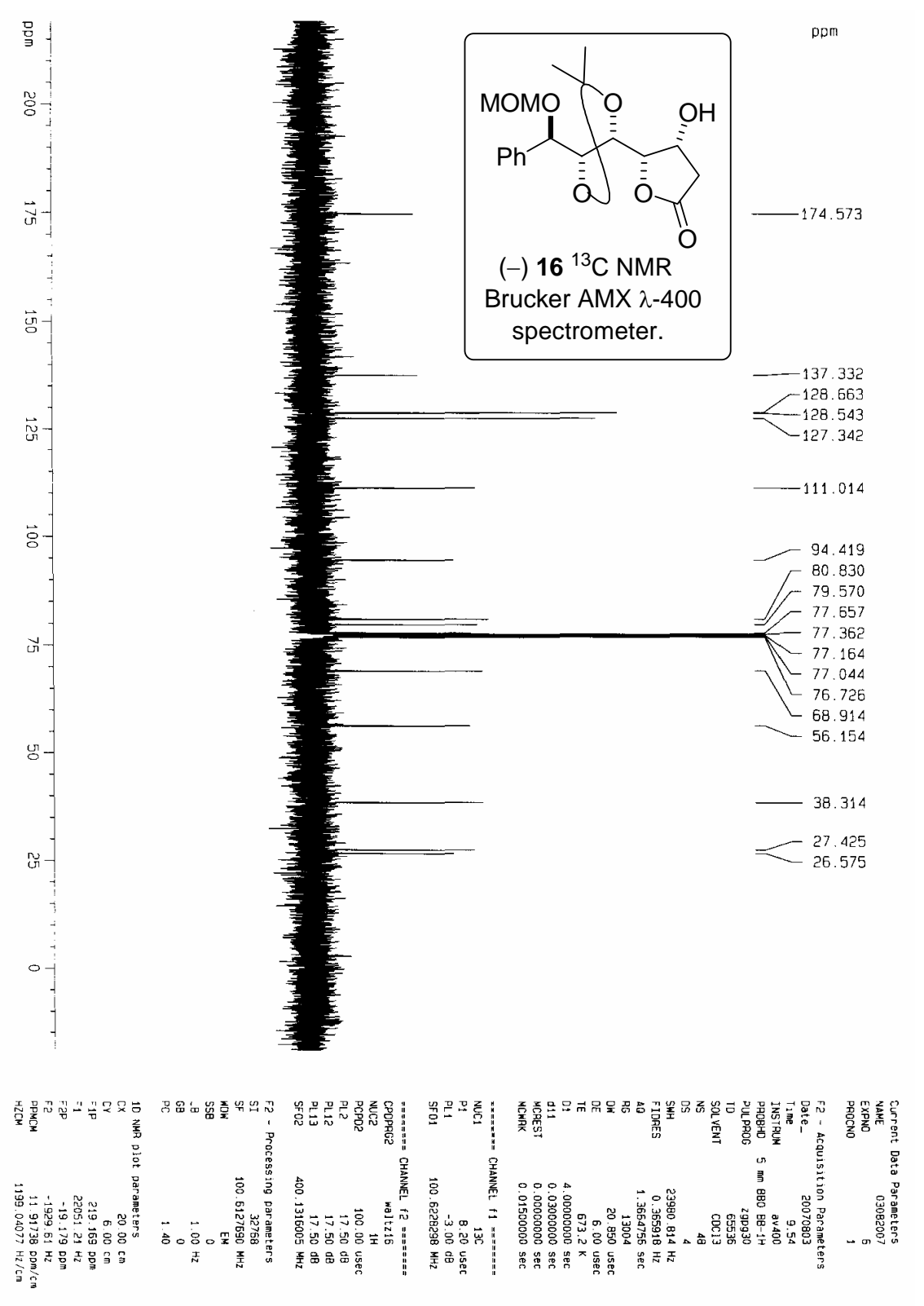




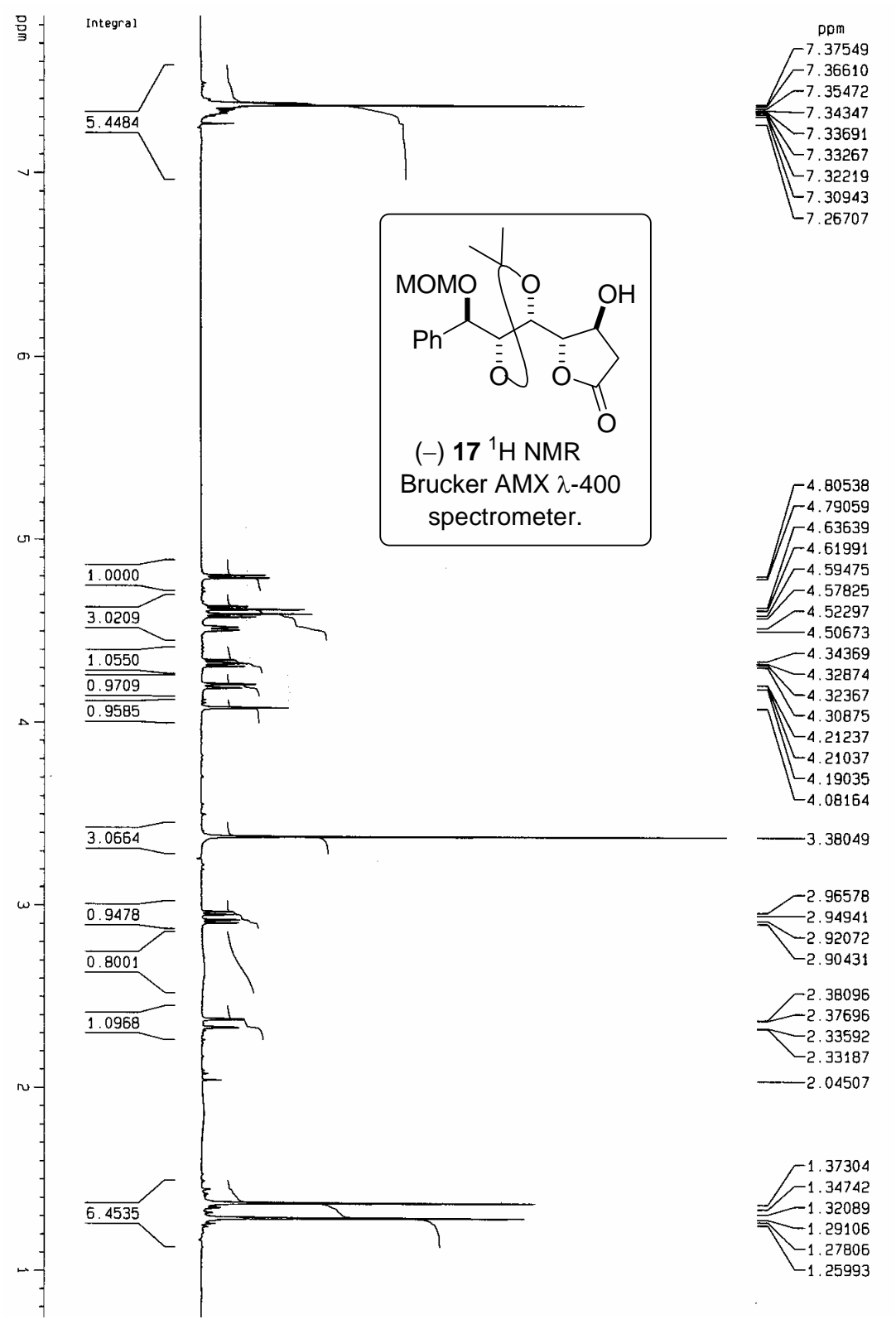



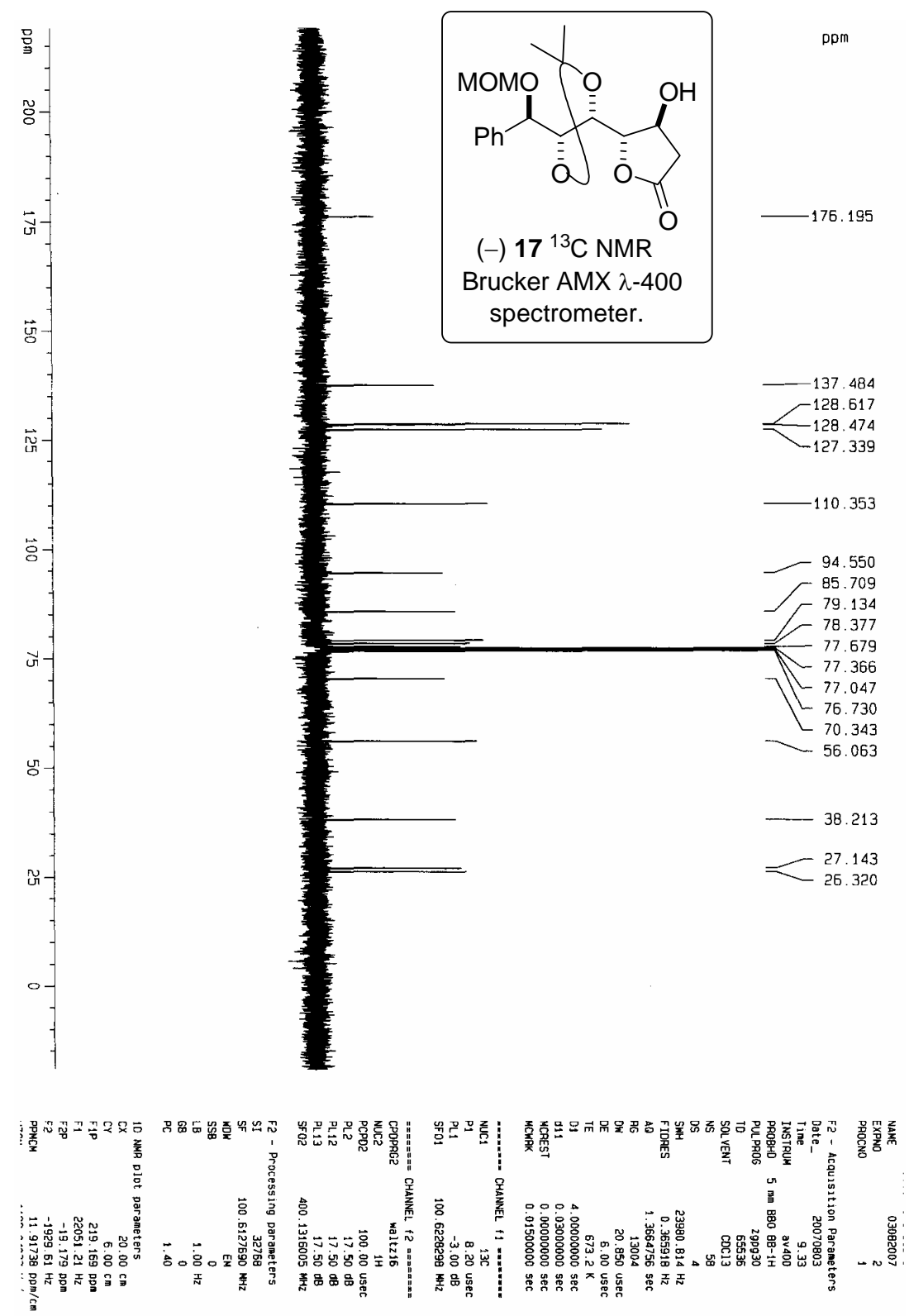


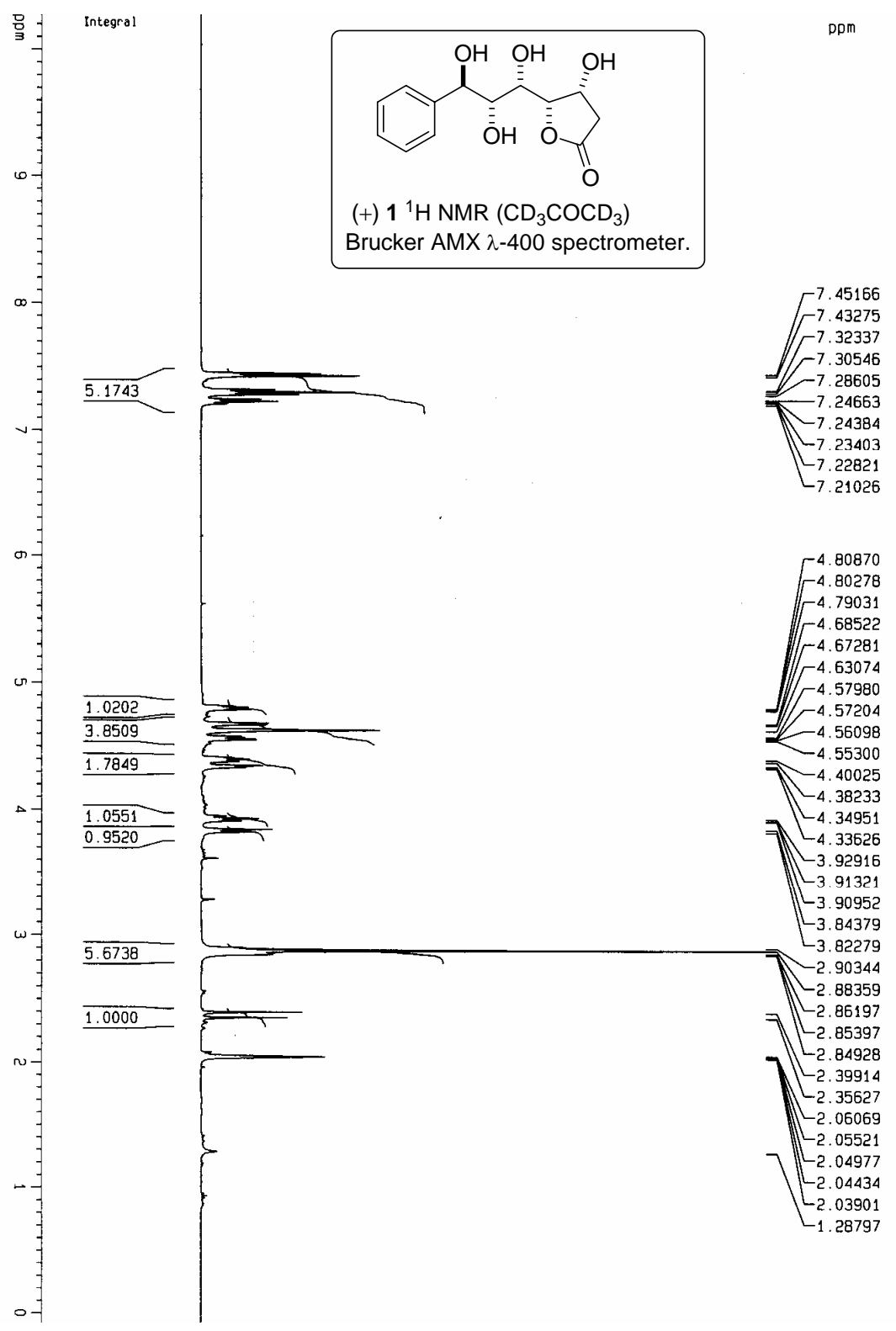




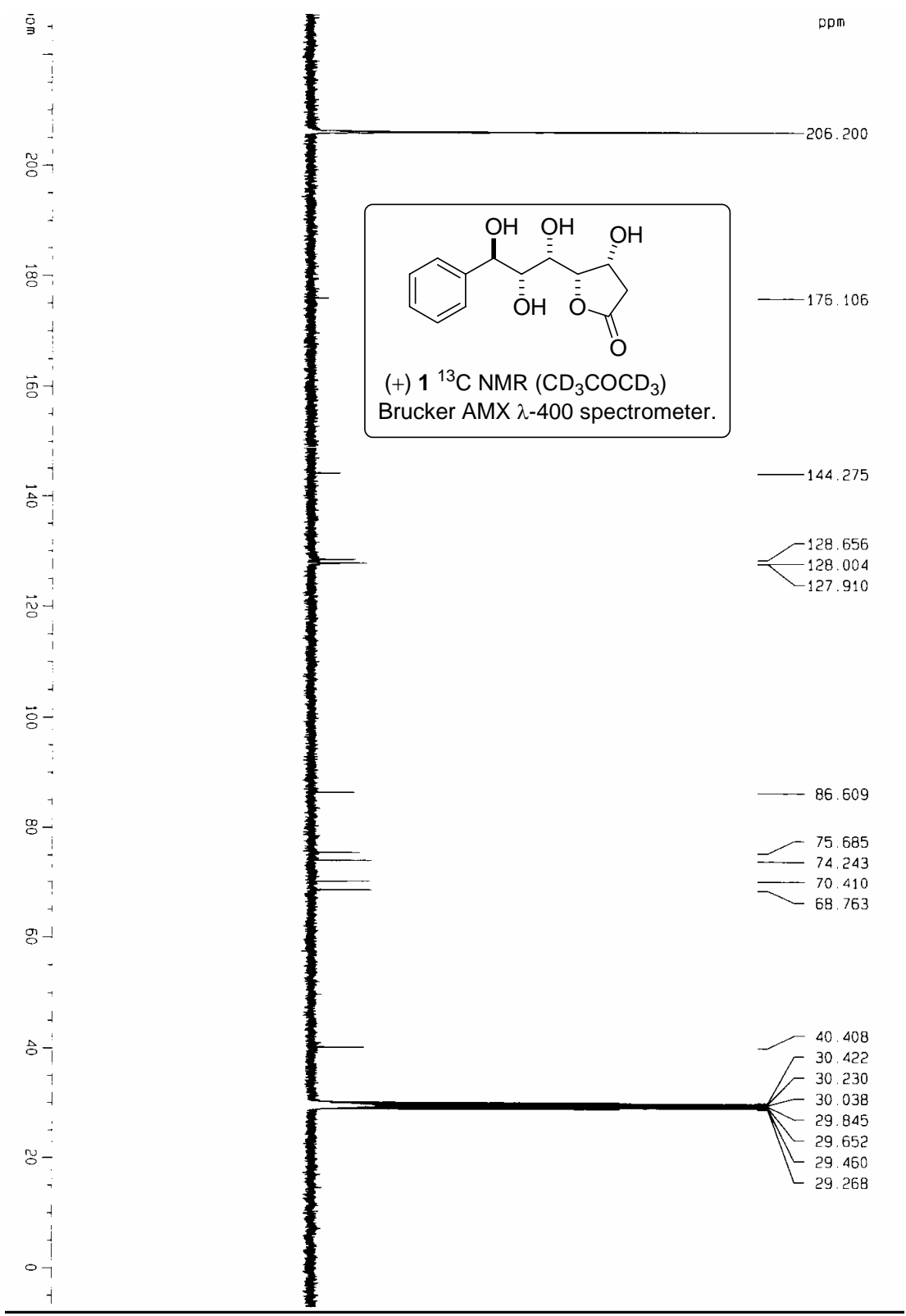




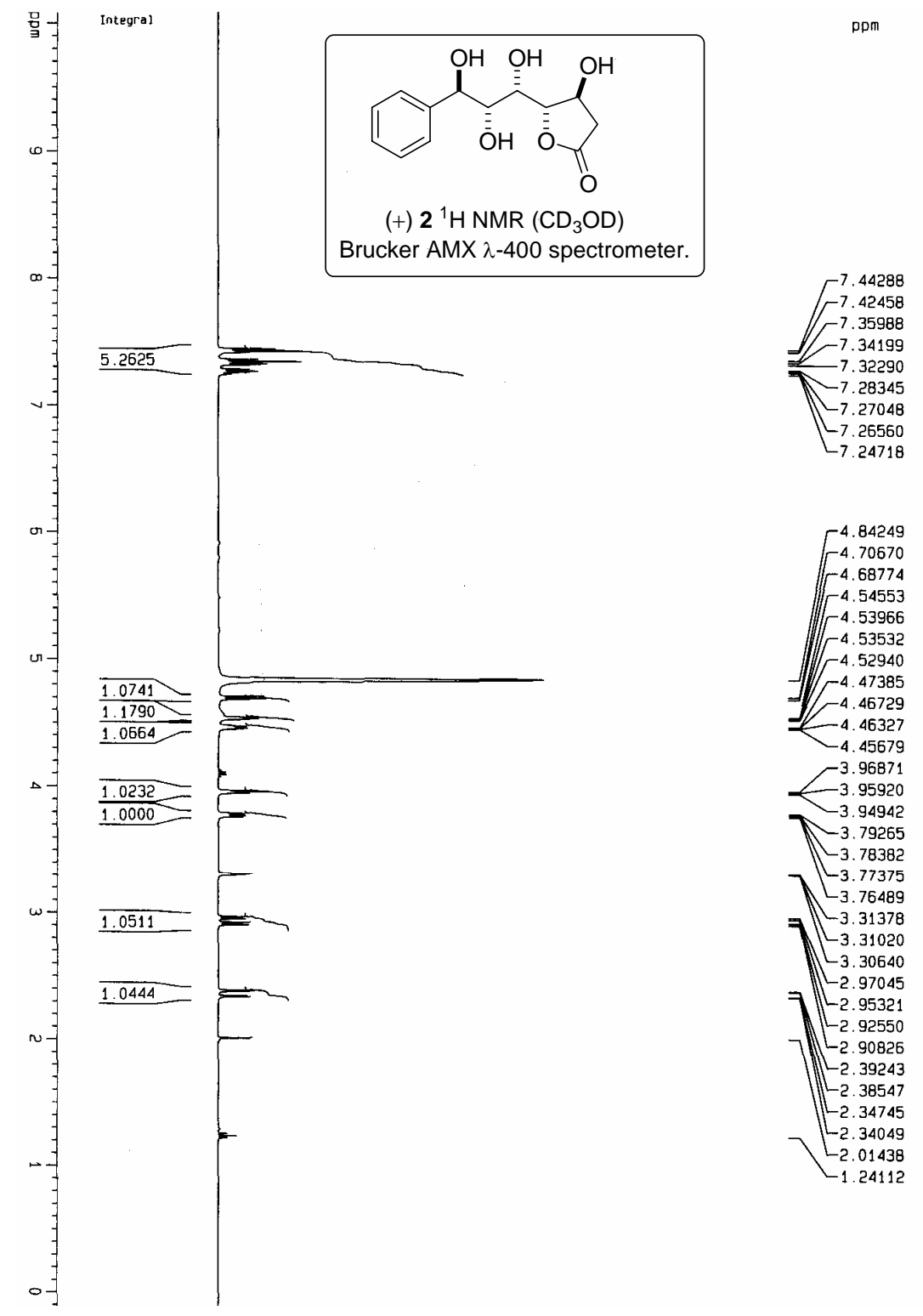




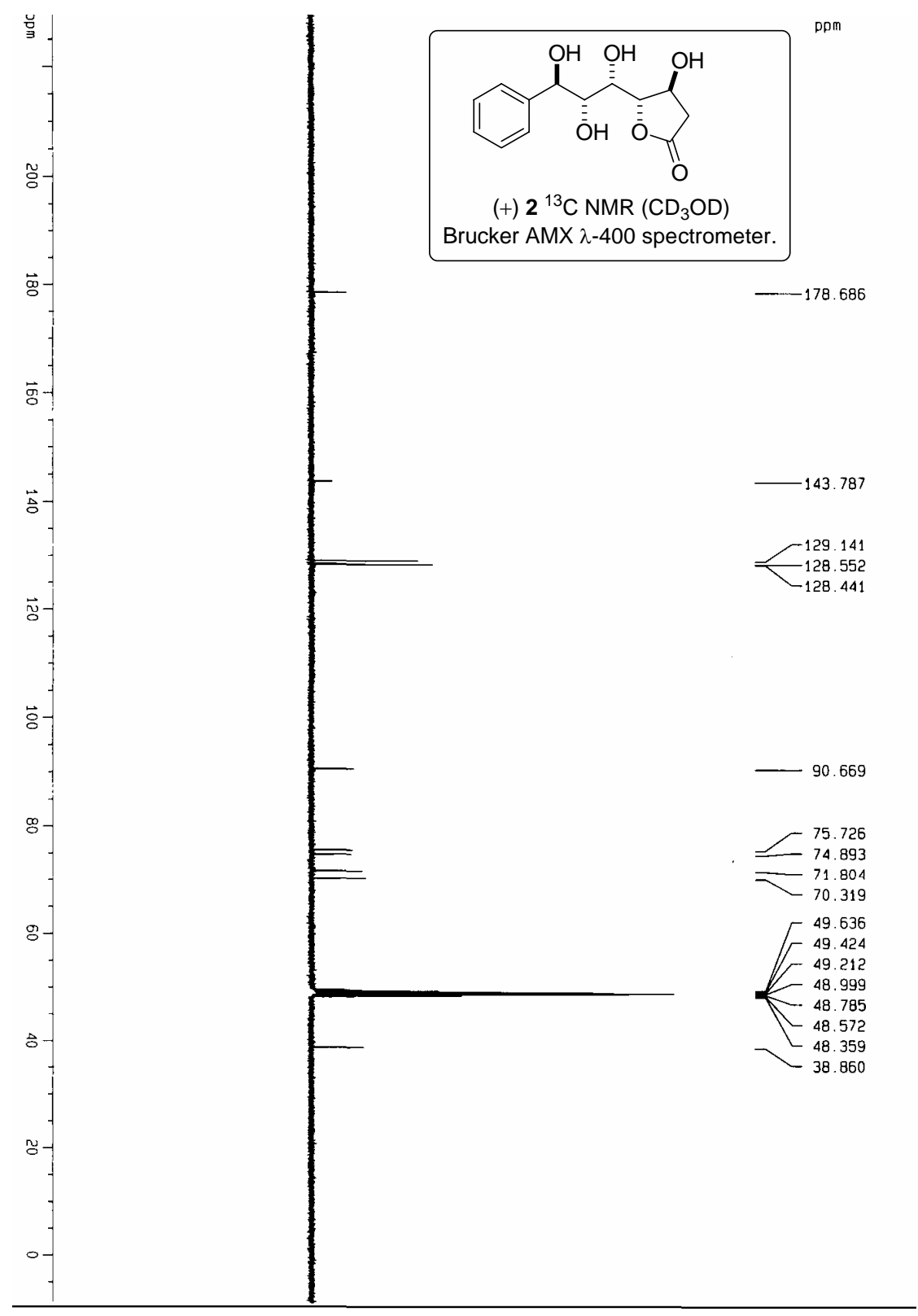




\section{Experimental:}

General Procedures: Column chromatography was performed on silica gel, Acme grade 100-200 mesh. TLC plates were visualized either with UV, in an iodine chamber, or with phosphomolybdic acid spray, unless noted otherwise. Unless stated otherwise, all reagents were purchased from commercial sources and used without additional purification. THF was freshly distilled over Na-benzophenone ketyl. Melting points were uncorrected. Unless stated otherwise, all the reactions were performed under inert atmosphere. Unless stated otherwise, all the NMR spectra were recorded in $\mathrm{CDCl}_{3}$. In ${ }^{13} \mathrm{C} \mathrm{NMR}$, the nature of the carbons $\left(\mathrm{C}, \mathrm{CH}, \mathrm{CH}_{2}, \mathrm{CH}_{3}\right)$ were determined by running the DEPT spectra.

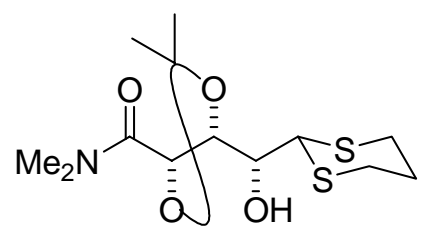

Preparation of $\quad(4 S, 5 R)-5-((R)-(1,3-d i t h i a n-2-y l)(h y d r o x y) m e t h y l)-N, N, 2,2-$ tetramethyl-1,3dioxolane-4-carboxamide (5): To a solution of 4 (1.8 g, $5.6 \mathrm{mmol})$ in methanol (20 mL) was added $\mathrm{CeCl}_{3} .7 \mathrm{H}_{2} \mathrm{O}(2.5 \mathrm{~g}, 6.7 \mathrm{mmol})$ and stirred at room temperature for $1 \mathrm{~h}$. The reaction mixture was cooled to $-78{ }^{\circ} \mathrm{C}$ and $\mathrm{NaBH}_{4}(0.26 \mathrm{~g}, 6.7 \mathrm{mmol})$ was added portion wise over a period of $30 \mathrm{~min}$ and stirred at the same temperature. After stirring for $0.5 \mathrm{~h}$, it was cautiously quenched by addition of water (15 mL) and extracted with EtOAc ( 3 x $20 \mathrm{~mL})$. The combined organic layers were washed with brine (30 mL), dried over $\mathrm{Na}_{2} \mathrm{SO}_{4}$ and concentrated. Silica gel column chromatography of the crude residue obtained after evaporation of the solvent, with petroleum ether:EtOAc (4:6) as eluent gave alcohol 5 (1.76 g, 97\%) as white crystalline solid. mp $134-136{ }^{\circ} \mathrm{C}$; $[\alpha]_{\mathrm{D}}+5.4$ (c 1.3, $\left.\mathrm{CHCl}_{3}\right)$; IR (KBr) 3428, 2908, 1637, 1505, 1380, 1220, 1158, $1073 \mathrm{~cm}^{-1} ;{ }^{1} \mathrm{H}$ NMR (300 MHz, $\left.\mathrm{CDCl}_{3}\right) \delta 5.07$ (d, $\left.J=7.8 \mathrm{~Hz}, 1 \mathrm{H}\right), 4.70(\mathrm{~d}, J=$ 7.8 Hz, 1H), 4.06-3.94 (m, 2H), 3.16 (s, 3H), 2.98 (s, 3H), 3.07-2.86 (m, 2H), 2.75-2.65 (m, 2H), 2.091.98 (m, 2H), 1.46 (s, 3H), 1.40 (s, 3H); ${ }^{13} \mathrm{C}$ NMR (75 MHz, CDCl $) \delta 168.5$ (C), 110.3 (C), 76.9 (CH), 
$74.6(\mathrm{CH}), 68.6(\mathrm{CH}), 47.6(\mathrm{CH}), 37.0\left(\mathrm{CH}_{3}\right), 35.6\left(\mathrm{CH}_{3}\right), 26.8\left(\mathrm{CH}_{2}\right), 26.5\left(\mathrm{CH}_{3}\right), 26.3\left(\mathrm{CH}_{2}\right), 25.3$ $\left(\mathrm{CH}_{2}\right)$; Analysis calcd for $\mathrm{C}_{13} \mathrm{H}_{23} \mathrm{NO}_{4} \mathrm{~S}_{2} \mathrm{C} 48.57 \mathrm{H}$ 7.21 N $4.36 \mathrm{~S} 19.95$; found C 48.63 H 7.02 N 4.37 S 19.72; HRMS for $\mathrm{C}_{13} \mathrm{H}_{23} \mathrm{NO}_{4} \mathrm{~S}_{2}+\mathrm{Na}$ calcd 344.0968; found 344.0966.

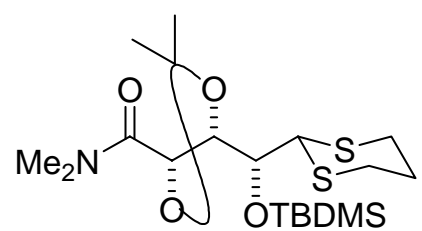

Preparation of $\quad(4 S, 5 S)-5-((R)-(1,3-d i t h i a n-2-y l)(t e r t-b u t y l d i m e t h y l s i l y l o x y) m e t h y l)-~ N, N, 2,2-$ tetramethyl-1,3-dioxolane-4-carboxamide (6): To a solution of alcohol 5 (0.84 g, $2.6 \mathrm{mmol})$ in dry DCM (12 mL) cooled to $0{ }^{\circ} \mathrm{C}$ were added, pyridine $(0.8 \mathrm{~mL}, 10.4 \mathrm{mmol})$, TBDMSOTf $(0.7 \mathrm{~mL}, 3.1$ mmol) and stirred for $1 \mathrm{~h}$ at the same temperature. The reaction mixture was quenched by addition of water $(10 \mathrm{~mL})$ and extracted with ether $(3 \mathrm{x} 15 \mathrm{~mL})$. Combined ethereal extracts were washed with $1 \mathrm{~N}$ $\mathrm{HCl}(10 \mathrm{~mL})$, brine $(20 \mathrm{~mL})$ and dried $\left(\mathrm{Na}_{2} \mathrm{SO}_{4}\right)$. Evaporation of the solvent and silica gel column chromatography of the residue using petroleum ether:EtOAc (7:3) as eluent afforded the corresponding silyl ether 6 (1.12 g, 98\%) as colorless oil. $[\alpha]_{\mathrm{D}}+10.9$ (c 1.1, $\left.\mathrm{CHCl}_{3}\right)$; IR (neat) 2931, 2856, 1654, 1255, 1132, 1070, $837 \mathrm{~cm}^{-1}$; ${ }^{1} \mathrm{H}$ NMR (300 MHz, $\left.\mathrm{CDCl}_{3}\right) \delta$ 4.82-4.71 (m, 2H), $4.31(\mathrm{~d}, J=4.5 \mathrm{~Hz}, 1 \mathrm{H}), 3.93$ (t, $J=4.5 \mathrm{~Hz}, 1 \mathrm{H}), 3.10$ (s, 3H), 2.90 (s, 3H), 2.87-2.72 (m, 4H), 2.05-1.97 (m, 1H), 1.88-1.78 (m, 1H), 1.41 (s, 3H), 1.36 (s, 3H), 0.87 (s, 9H), 0.14 (s, 3H), 0.03 (s, 3H); ${ }^{13} \mathrm{C}$ NMR $\left(75 \mathrm{MHz}, \mathrm{CDCl}_{3}\right) \delta 168.8$ (C), $110.3(\mathrm{C}), 79.2(\mathrm{CH}), 74.5(\mathrm{CH}), 74.0(\mathrm{CH}), 51.0(\mathrm{CH}), 37.0\left(\mathrm{CH}_{3}\right), 35.8\left(\mathrm{CH}_{3}\right), 30.9\left(\mathrm{CH}_{2}\right), 30.1$ $\left(\mathrm{CH}_{2}\right), 26.8\left(\mathrm{CH}_{3}\right), 26.1\left(\mathrm{CH}_{3}\right), 25.8\left(\mathrm{CH}_{3}\right), 18.3(\mathrm{C}),-4.4\left(\mathrm{CH}_{3}\right),-4.5\left(\mathrm{CH}_{3}\right)$; HRMS for $\mathrm{C}_{19} \mathrm{H}_{37} \mathrm{NO}_{4} \mathrm{~S}_{2} \mathrm{Si}+\mathrm{Na}$ calcd 458.1833; found 458.1831. 


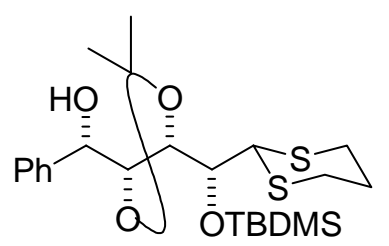

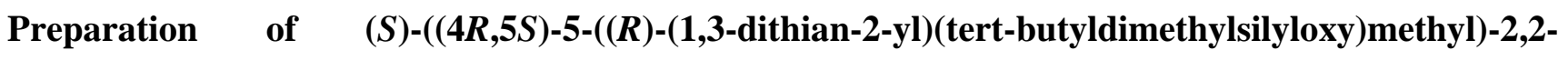
dimethyl-1,3-dioxolan-4-yl)(phenyl)methanol (8): To a pre cooled solution $\left(-78{ }^{\circ} \mathrm{C}\right)$ of 7 (0.75 g, 1.6 mmol) in dry THF $(10 \mathrm{~mL})$ was added K-selectride $(1.9 \mathrm{~mL}$ of $1 \mathrm{M}$ solution in THF, $1.9 \mathrm{mmol})$ dropwise under argon atmosphere. The reaction mixture was stirred for $1 \mathrm{~h}$ at the same temperature. After the reaction was complete (TLC), it was allowed to warm to $0{ }^{\circ} \mathrm{C}$. The reaction mixture was quenched by cautious addition of water ( $8 \mathrm{~mL}$ ) and stirred for $30 \mathrm{~min}$. It was then extracted with ether (3 x $20 \mathrm{~mL}$ ) and the combined ethereal extracts were washed with brine $(25 \mathrm{~mL})$ and dried over $\mathrm{Na}_{2} \mathrm{SO}_{4}$. Evaporation of the solvent and silica gel column chromatography of the residue using petroleum ether:EtOAc (95:5) as eluent gave diastereomeric mixture ( $d r$ 92:8) of alcohols with 8 being the major isomer (0.66 g, 88\%) as white crystalline solid. mp 102-103 ${ }^{\circ} \mathrm{C}$; $[\alpha]_{\mathrm{D}}+21.2\left(\right.$ c $\left.1, \mathrm{CHCl}_{3}\right)$; IR (KBr) 3508, 2933, 1468, 1380, 1252, 1128, 1056, 883, $778 \mathrm{~cm}^{-1}$; ${ }^{1} \mathrm{H}$ NMR (400 MHz, $\left.\mathrm{CDCl}_{3}\right) \delta$ 7.48-7.25 (m, 5H), $4.77(\mathrm{t}, J=4.7 \mathrm{~Hz}, 1 \mathrm{H}), 4.32-4.21(\mathrm{~m}, 2 \mathrm{H}), 4.00(\mathrm{~d}, J=3.8 \mathrm{~Hz}, 1 \mathrm{H}), 3.44(\mathrm{t}, J=3.6 \mathrm{~Hz}, 1 \mathrm{H}), 2.97(\mathrm{~d}, J=$ 5.2 Hz, 1H), 2.79-2.56 (m, 4H), 2.07-1.93 (m, 1H), 1.88-1.76 (m, 1H), 1.47 (s, 3H), 1.44 (s, 3H), 0.94 (s, 9H), 0.11 (s, 3H), 0.01 (s, 3H); ${ }^{13} \mathrm{C}$ NMR (100 MHz, $\left.\mathrm{CDCl}_{3}\right) \delta 140.4(\mathrm{C}), 128.6$ (CH), $128.1(\mathrm{CH}), 127.0$ (CH), $110.1(\mathrm{C}), 81.2(\mathrm{CH}), 78.8(\mathrm{CH}), 74.8(\mathrm{CH}), 74.5(\mathrm{CH}), 52.6(\mathrm{CH}), 31.2\left(\mathrm{CH}_{2}\right), 30.5\left(\mathrm{CH}_{2}\right), 27.8$ $\left(\mathrm{CH}_{3}\right), 27.5\left(\mathrm{CH}_{3}\right), 26.2\left(\mathrm{CH}_{2}\right), 26.1\left(\mathrm{CH}_{3}\right), 18.6(\mathrm{C}),-4.0\left(\mathrm{CH}_{3}\right),-4.4\left(\mathrm{CH}_{3}\right)$; Analysis calcd for $\mathrm{C}_{23} \mathrm{H}_{38} \mathrm{O}_{4} \mathrm{~S}_{2} \mathrm{Si}$ C $58.68 \mathrm{H}$ 8.14 S 13.62; found C $58.62 \mathrm{H}$ 7.97 S 13.82; HRMS for $\mathrm{C}_{23} \mathrm{H}_{38} \mathrm{O}_{4} \mathrm{~S}_{2} \mathrm{Si}+\mathrm{Na}$ calcd 493.1881; found 493.1879. 


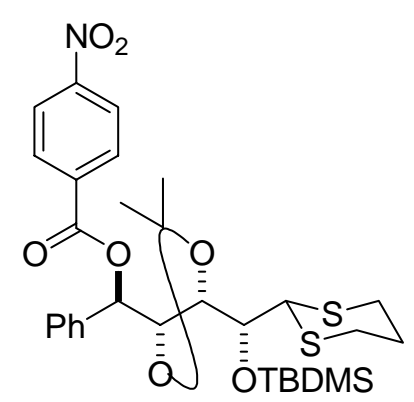

mp 74.3-76.5 ${ }^{\circ} \mathrm{C} ;[\alpha]_{\mathrm{D}}+43.3\left(\right.$ (c 1.2, $\left.\mathrm{CHCl}_{3}\right)$; IR (KBr) 2955, 1716, 1608, 1532, 1346, 1277, 1135, 851, $718 \mathrm{~cm}^{-1}$; ${ }^{1} \mathrm{H}$ NMR (400 MHz, $\left.\mathrm{CDCl}_{3}\right) \delta$ 8.33-8.26 (m, 4H), 7.62 (d, $\left.J=7.2 \mathrm{~Hz}, 2 \mathrm{H}\right), 7.45-7.30$ (m, 3H), $6.42(\mathrm{~d}, J=2.1 \mathrm{~Hz}, 1 \mathrm{H}), 4.50$ (d, $J=2.8 \mathrm{~Hz}, 1 \mathrm{H}), 4.42$ (dd, $J=7.8,2.3 \mathrm{~Hz}, 1 \mathrm{H}), 4.26$ (dd, $J=7.8,5.6$ Hz, 1H), 3.74 (dd, $J=5.4,2.9$ Hz, 1H), 3.12-2.99 (m, 2H), 2.91-2.79 (m, 2H), 2.19-2.08 (m, 1H), 2.031.85 (m, 1H), 1.37 (s, 3H), 0.94 (s, 9H), 0.92 (s, 3H), 0.12 (s, 3H), 0.04 (s, 3H); ${ }^{13} \mathrm{C}$ NMR (100 MHz, $\left.\mathrm{CDCl}_{3}\right) \delta 164.0(\mathrm{C}), 150.7(\mathrm{C}), 135.4(\mathrm{C}), 134.7(\mathrm{C}), 131.0(\mathrm{CH}), 128.8(\mathrm{CH}), 128.7(\mathrm{CH}), 128.3(\mathrm{CH})$, 123.6 (CH), $110.6(\mathrm{C}), 79.4(\mathrm{CH}), 79.1(\mathrm{CH}), 76.8(\mathrm{CH}), 53.2(\mathrm{CH}), 31.6\left(\mathrm{CH}_{2}\right), 30.5\left(\mathrm{CH}_{2}\right), 27.5\left(\mathrm{CH}_{3}\right)$, $26.7\left(\mathrm{CH}_{3}\right), 26.3\left(\mathrm{CH}_{2}\right), 26.1\left(\mathrm{CH}_{3}\right), 18.6(\mathrm{C}),-3.8\left(\mathrm{CH}_{3}\right),-4.6\left(\mathrm{CH}_{3}\right)$; Analysis calcd for $\mathrm{C}_{30} \mathrm{H}_{41} \mathrm{NO}_{7} \mathrm{~S}_{2} \mathrm{Si}$ C 58.13 H 6.67 N 2.26 S 10.35; found C 58.12 H 6.64 N 2.69 S 10.08; HRMS for $\mathrm{C}_{30} \mathrm{H}_{41} \mathrm{NO}_{7} \mathrm{~S}_{2} \mathrm{Si}+\mathrm{Na}$ calcd 642.1994; found 642.1991.

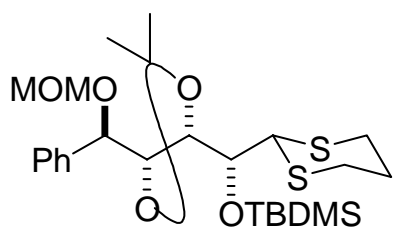

Preparation of $\quad((R)-(1,3-d i t h i a n-2-y l)((4 S, 5 R)-5-((R)-(m e t h o x y m e t h o x y)(p h e n y l) m e t h y l)-2,2-$ dimethyl-1,3-dioxolan-4-yl)methoxy)(tert-butyl)dimethylsilane (10): To a solution of alcohol 9 (0.52 g, $1.0 \mathrm{mmol})$ in dry DCM $(8.0 \mathrm{~mL})$ were added diisopropylethyl amine $(0.5 \mathrm{~g}, 0.7 \mathrm{~mL}, 4.0 \mathrm{mmol})$, DMAP (24 mg, $20 \mathrm{~mol} \%)$ and $\mathrm{MOMCl}(0.11 \mathrm{~mL}, 1.5 \mathrm{mmol})$ at room temperature. After stirring for 15 min at room temperature, the reaction mixture was refluxed $10 \mathrm{~h}$. It was cooled to room temperature, quenched by addition of water (10 mL). It was then extracted with ether ( 3 x $15 \mathrm{~mL}$ ). Combined ethereal 
extracts were washed with brine $(20 \mathrm{~mL})$ and dried $\left(\mathrm{Na}_{2} \mathrm{SO}_{4}\right)$. Evaporation of the solvent and silica gel column chromatography of the residue using petroleum ether:EtOAc (95:5) as eluent yielded $\mathbf{1 0}$ (0.55 g, 97\%) as a colorless oil. $[\alpha]_{\mathrm{D}}-42.4\left(c 1, \mathrm{CHCl}_{3}\right)$; IR (neat) 2934, 1598, 1467, 1373, 1251, 1134, 1098, 1029, $837 \mathrm{~cm}^{-1}$; ${ }^{1} \mathrm{H}$ NMR (400 MHz, $\mathrm{CDCl}_{3}$ ) $\delta$ 7.38-7.19 (m, 5H), 4.72 (d, $\left.J=3.9 \mathrm{~Hz}, 1 \mathrm{H}\right), 4.52-4.46$ (m, 2H), 4.23-4.16 (m, 3H), 3.67 (t, $J=3.7 \mathrm{~Hz}, 1 \mathrm{H}), 3.33$ (s, 3H), 2.83-2.74 (m, 4H), 2.05-1.75 (m, 2H), 1.31 (s, 3H), 1.11 (s, 3H), 0.86 (s, 9H), 0.06 (s, 3H), 0.01 (s, 3H); ${ }^{13} \mathrm{C}$ NMR (100 MHz, $\left.\mathrm{CDCl}_{3}\right) \delta 137.3$ (C), $128.7(\mathrm{CH}), 128.2(\mathrm{CH}), 110.1(\mathrm{C}), 94.1(\mathrm{CH}), 80.1(\mathrm{CH}), 79.4(\mathrm{CH}), 77.9(\mathrm{CH}), 75.7(\mathrm{CH}), 56.0$ $\left(\mathrm{CH}_{3}\right), 53.4(\mathrm{CH}), 31.5\left(\mathrm{CH}_{2}\right), 30.8\left(\mathrm{CH}_{2}\right), 27.4\left(\mathrm{CH}_{3}\right), 27.2\left(\mathrm{CH}_{3}\right), 26.3\left(\mathrm{CH}_{2}\right), 26.1\left(\mathrm{CH}_{3}\right), 18.6(\mathrm{C})$, -3.7 $\left(\mathrm{CH}_{3}\right),-4.3\left(\mathrm{CH}_{3}\right)$; HRMS for $\mathrm{C}_{25} \mathrm{H}_{42} \mathrm{O}_{5} \mathrm{~S}_{2} \mathrm{Si}+\mathrm{Na}$ calcd 537.2143; found 537.2141.

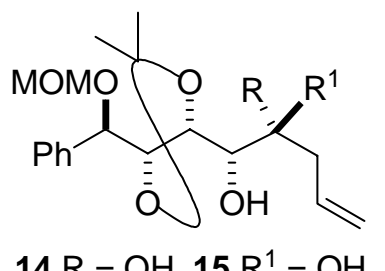

Preparation of (1S)-1-((4S,5R)-5-((S)-(methoxymethoxy)(phenyl)methyl)-2,2-dimethyl-1,3dioxolan-4-yl)-4-ene-1,2-diols (14 and 15): To a pre cooled $\left(0^{\circ} \mathrm{C}\right)$ solution of alcohols 12 and $13(0.2$ g, $0.43 \mathrm{mmol})$ in dry THF (5.0 mL) was added TBAF (1M solution in THF, $1.0 \mathrm{~mL}, 1.0 \mathrm{mmol}$ ) under argon atmosphere. The reaction mixture was slowly allowed to warm up to room temperature and stirred for $0.5 \mathrm{~h}$ at room temperature. Water $(10 \mathrm{~mL})$ was added to the reaction mixture and stirred for $10 \mathrm{~min}$. It was then extracted with ether ( 3 x $10 \mathrm{~mL})$. Combined ethereal extracts were washed with brine (15 $\mathrm{mL}$ ) and dried over $\mathrm{Na}_{2} \mathrm{SO}_{4}$. Evaporation of solvent and silica gel column chromatography of the residue using petroleum ether:EtOAc (6:4) as eluent yielded diastereomeric mixture of diols 14 and $\mathbf{1 5}$ (0.15 g, 97\%) as a colorless oil. IR (neat) 3469, 2926, 1641, 1455, 1380, 1247, 1215, 1153, 1035, 917, $702 \mathrm{~cm}^{-1}$; ${ }^{1} \mathrm{H}$ NMR (400 MHz, $\left.\mathrm{CDCl}_{3}\right) \delta$ 7.37-7.28 (m, 5H), 5.88-5.67 (m, 1H), 5.23-5.05 (m, 2H), 4.82-4.75 (m, 
1H), 4.65-4.52 (m, 2H), 4.42-4.33 (m, 1.5H), 4.18-4.09 (m, 0.5H), 3.82-3.61 (m, 2H), 3.38-3.35 (m, 3H), 3.18-3.08 (m, 1H), 2.62-2.08 (m, 2H), 1.38 (S, 6H); ${ }^{13} \mathrm{C}$ NMR (100 MHz, $\left.\mathrm{CDCl}_{3}\right) \delta$ 137.7, 137.6, 134.4, 134.3, 128.5, 128.4, 128.3, 127.7, 127.5, 118.2, 117.8, 116.1, 110.0, 109.8, 94.5, 80.6, 79.9, 77.8, 77.7, 77.5, 72.9, 72.7, 71.4, 70.3, 56.0, 55.9, 38.5, 37.6, 27.2, 27.1, 27.0 ; HRMS for $\mathrm{C}_{19} \mathrm{H}_{28} \mathrm{O}_{6}+\mathrm{Na}$ calcd 375.1786; found 375.1784.

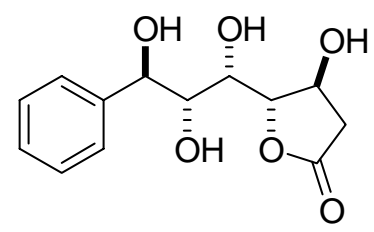

Preparation of (+)-3-epi-Cardiobutanolide (2): 2 was synthesized using a procedure similar to that described for the synthesis of 1. $[\alpha]_{D}+27.6\left(c\right.$ 1.0, MeOH) $\left[\operatorname{lit}^{4 c}[\alpha]_{D}+26.5(c 0.7, \mathrm{MeOH})\right]$; IR (neat) 3384, 1768, 1677, 1453, 1202, $1024 \mathrm{~cm}^{-1}$; ${ }^{1} \mathrm{H}$ NMR (400 MHz, CD $\left.{ }_{3} \mathrm{OD}\right) \delta 7.43$ (d, $J=7.2 \mathrm{~Hz}, 2 \mathrm{H}$ ), 7.34 (t, $J=7.2 \mathrm{~Hz}, 2 \mathrm{H}), 7.27$ (t, $J=7.2 \mathrm{~Hz}, 1 \mathrm{H}), 4.69$ (d, $J=7.6 \mathrm{~Hz}, 1 \mathrm{H}$, ), 4.53 (dd, $J=4.1,2.3 \mathrm{~Hz}, 1 \mathrm{H}), 4.47$ (td, $J=6.8,2.5 \mathrm{~Hz}, 1 \mathrm{H}), 3.95$ (t, $J=3.8 \mathrm{~Hz}, 1 \mathrm{H}), 3.78$ (dd, $J=7.6,3.5 \mathrm{~Hz}, 1 \mathrm{H}), 2.94$ (dd, $J=18.0,6.9$ Hz, 1H), 2.36 (dd, $J=18.0,2.8 \mathrm{~Hz}, 1 \mathrm{H}) ;{ }^{13} \mathrm{C}$ NMR (100 MHz, CD $\left.3 \mathrm{OD}\right) \delta 178.7$ (C), $143.8(\mathrm{C}), 129.1$ (CH), $128.6(\mathrm{CH}), 128.4(\mathrm{CH}), 90.7(\mathrm{CH}), 75.7(\mathrm{CH}), 74.9(\mathrm{CH}), 71.8(\mathrm{CH}), 70.3(\mathrm{CH}), 38.9\left(\mathrm{CH}_{2}\right)$; HRMS for $\mathrm{C}_{13} \mathrm{H}_{16} \mathrm{O}_{6}+\mathrm{Na}$ calcd 291.0847; found 291.0845. 